\title{
On the Generalization of Natural Type Selection to Multiple Description Coding
}

\author{
Yuhua Fan, Jia Wang, Jun Sun, Member, IEEE, and Jun Chen, Member, IEEE
}

\begin{abstract}
Natural type selection was originally proposed by Zamir and Rose for universal single description coding. In this paper, we generalize this principle to universal multiple description coding (MDC). Two schemes based on random codebooks are proposed: one is of fixed distortion and the other is of fixed weight. Their operational sum-rate-distortion functions are derived, which coincide with the EGC (El GamalCover) sum-rate bound if the parameters of the schemes are optimized. It is also shown that in both schemes the joint type of reconstruction codewords can be used to improve the ratedistortion (R-D) performance. Based on our theoretical results, a practical universal scheme is proposed by leveraging the MDC methods based on low-density generator matrix (LDGM) codes. The performance of this scheme is compared experimentally with the EGC bound, which shows its effectiveness.
\end{abstract}

Index Terms-Data compression, natural type selection, universal multiple description coding.

\section{INTRODUCTION}

W E study the problem of universal multiple description coding (UMDC), in which no knowledge of the source statistics is assumed, while good operational rate-distortion (R-D) performance is still expected asymptotically. Although significant effort has been devoted to universal lossless/lossy data compression, research on UMDC is still very limited. It is worth noting that the techniques used in designing universal lossy single description codes are not directly applicable to UMDC due to the fact that there are multiple decoders in MDC and each decoder decodes a different set of descriptions.

As pointed out in [3], since a lossy decoder only needs to produce an approximate replica of the source, the aim of its codebook adaptation is not to match itself to the source statistics but to find the "right" set of type classes. Furthermore, a mechanism of natural type selection is proposed in [3] for lossy single description coding. It is shown that, given

Manuscript received December 7, 2011; revised June 2, September 22, and November 9, 2012. The associate editor coordinating the review of this paper and approving it for publication was Z Xiong.

Y. Fan, J. Wang, and J. Sun are with the Department of Electronic Engineering, Shanghai Jiao Tong University, and the Shanghai Key Laboratory of Digital Media Processing and Transmission, Shanghai, China (e-mail: \{sjtufyh, jiawang, junsun\}@ sjtu.edu.cn).

J. Chen is with the Department of Electrical and Computer Engineering, McMaster University, Hamilton, ON L8S 4K1, Canada (e-mail: junchen@mail.ece.mcmaster.ca).

Y. Fan and J. Wang were supported in part by the NSFC under Grants 61271221 and U1201255, and the National Key Technology R\&D Program of China under Grant 2013BAH53F04.

J. Chen was supported in part by the Early Researcher Award from the Province of Ontario and in part by the Natural Science and Engineering Research Council (NSERC) of Canada under a Discovery Grant.

The material in this paper was presented in part at the Data Compression Conference, Snowbird, UT, March 2011.

Digital Object Identifier 10.1109/TCOMM.2012.021513.110833 a codebook, codewords of certain type will be favored during the coding process, based on which a better codebook can be generated. The theory of natural type selection illuminates the connection among universal source coding, Blahut-Arimoto algorithm, and the rate distortion theory.

In this paper, we generalize the concept of natural type selection to two-channel MDC, in which the source is encoded into two descriptions and each description is either received entirely or lost completely. We propose two schemes based on random codebooks: one is of fixed-distortion and the other is of fixed-weight. For both schemes, we analyze the relationship between sum-rate and distortion, and establish results that are parallel to those given in [3]. It is shown that the R-D performance of the proposed schemes improves progressively as coding proceeds. Inspired by these theoretical results, we then propose a practical universal scheme by leveraging the MDC methods based on low-density generator matrix (LDGM) codes [12]. Experimental results show that the R-D performance of this scheme approaches the El GamalCover (EGC) bound.

Recent work on UMDC includes [1], [2]. In [1], MDC of discrete ergodic sources is studied. The relationship between block MDC and stationary MDC is revealed, based on which a universal coding algorithm is proposed. Given an $n$ dimensional source vector $\mathbf{x} \in \mathcal{X}^{n}$, a triple of $n$-dimensional vectors $(\mathbf{y}, \mathbf{z}, \mathbf{w})$ is first found by solving an optimization problem with simulated annealing, after which $\mathbf{y}$ and $\mathbf{z}$ are compressed separately by the Lempel-Ziv algorithm, and $\mathbf{w}$ is compressed losslessly conditioned on both $\mathbf{y}$ and $\mathbf{z}$. In [2], a universal scheme is presented for multiple description scalar quantization under the assumption that the cells of side quantizers are Voronoi regions. It is also shown that the complexity of the scheme can be greatly reduced by exploiting the structure of quantizers.

The rest of the paper is organized as follows. Section II introduces the notations and conventions used throughout the paper. Section III contains the definition of the EGC region along with a discussion of its properties. Section IV presents two UMDC schemes based on random codebooks, supported by several theoretical results. A practical UMDC scheme is proposed in Section $\mathrm{V}$, and the experimental results are given in Section VI. We conclude the paper in Section VII.

\section{NOTATIONS AND CONVENTIONS}

We use uppercase letters (e.g., $U$ ) to denote random variables, use their lowercase counterparts (e.g., $u$ ) to denote their realizations, and use calligraphic letters (e.g., $\mathcal{U}$ ) to denote the corresponding alphabets. Lowercase boldface letters (e.g., 
u) represent deterministic vectors while uppercase boldface letters (e.g., U) represent random vectors or matrices. Given an $n$-dimensional vector $\mathbf{u} \in \mathcal{U}^{n}$, its $i$-th element is denoted by $\mathbf{u}[i]$, and its type is denoted by $t_{\mathbf{u}}(u), u \in \mathcal{U}$. We use $t_{\mathbf{u v}}(\cdot)$ for the joint type of $\mathbf{u}$ and $\mathbf{v}$. Given a matrix $\mathbf{A}$, we use $\mathbf{A}[i][j]$ to denote its $(i, j)$ entry.

We assume that the source is an i.i.d. process generated by a generic random variable $X$. We denote the source alphabet by $\mathcal{X}$, and the probability mass function (pmf) of $X$ by $p_{X}(x), x \in \mathcal{X}$. Given two alphabets $\mathcal{U}$ and $\mathcal{V}$, the distortion between $u \in \mathcal{U}$ and $v \in \mathcal{V}$ is represented by $d_{\mathcal{U}, \mathcal{V}}(u, v)$. For $n$-dimensional vectors $\mathbf{u} \in \mathcal{U}^{n}$ and $\mathbf{v} \in \mathcal{V}^{n}$, we define $d_{\mathcal{U}, \mathcal{V}}(\mathbf{u}, \mathbf{v}) \triangleq \frac{1}{n} \sum_{i=1}^{i=n} d_{\mathcal{U}, \mathcal{V}}(\mathbf{u}[i], \mathbf{v}[i])$. For simplicity, $d_{\mathcal{U}, \mathcal{V}}(\cdot, \cdot)$ is abbreviated to $d(\cdot, \cdot)$ if the underlying alphabets can be inferred from the context. The logarithm function is to base 2 throughout the paper.

\section{Some BAsic Results on the EGC Region}

In two-channel MDC, a quintuple $\left(R_{1}, R_{2}, D_{0}, D_{1}, D_{2}\right)$ is achievable if for every $\epsilon>0$, there exist two encoding functions $\psi_{1}: \mathcal{X}^{n} \rightarrow\left\{1,2, \cdots, M_{1}\right\}, \psi_{2}$ : $\mathcal{X}^{n} \rightarrow\left\{1,2, \cdots, M_{2}\right\}$, and three decoding functions $\phi_{1}$ : $\left\{1,2, \cdots, M_{1}\right\} \rightarrow \mathcal{X}_{1}^{n}, \phi_{2}:\left\{1,2, \cdots, M_{2}\right\} \rightarrow \mathcal{X}_{2}^{n}, \phi_{0}:$ $\left\{1,2, \cdots, M_{1}\right\} \times\left\{1,2, \cdots, M_{2}\right\} \rightarrow \mathcal{X}_{0}^{n}$, such that

$$
\begin{aligned}
& \frac{1}{n} \log \left(M_{i}\right) \leq R_{i}+\epsilon, \\
& E\left[d\left(\mathbf{X}, \phi_{i}\left(\psi_{i}(\mathbf{X})\right)\right)\right] \leq D_{i}+\epsilon, i=1,2, \\
& E\left[d\left(\mathbf{X}, \phi_{0}\left(\psi_{1}(\mathbf{X}), \psi_{2}(\mathbf{X})\right)\right)\right] \leq D_{0}+\epsilon,
\end{aligned}
$$

where $\mathbf{X}$ is an $n$-dimensional random source vector.

The EGC theorem [6] states that for two-channel MDC a quintuple $\left(R_{1}, R_{2}, D_{0}, D_{1}, D_{2}\right)$ is achievable if $\left(R_{1}, R_{2}, D_{0}, D_{1}, D_{2}\right) \in \operatorname{conv}(\mathscr{A})$, where

$$
\begin{aligned}
\mathscr{A} \triangleq\{ & \left(R_{0}, R_{1}, D_{0}, D_{1}, D_{2}\right): \exists \text { random variables } X_{i} \in \mathcal{X}_{i} \\
& i=0,1,2, \text { such that } R_{i} \geq I\left(X ; X_{i}\right), i=1,2, \\
& R_{1}+R_{2} \geq I\left(X ; X_{0}, X_{1}, X_{2}\right)+I\left(X_{1} ; X_{2}\right) \\
& \left.E\left[d\left(X, X_{i}\right)\right] \leq D_{i}, i=0,1,2\right\}
\end{aligned}
$$

and $\operatorname{conv}(\mathscr{A})$ denotes the convex hull of $\mathscr{A}$. Define

$$
\begin{aligned}
& \widetilde{\mathscr{A}}_{\text {sum }} \triangleq\left(R, D_{0}, D_{1}, D_{2}\right): \exists\left(R_{1}, R_{2}, D_{0}, D_{1}, D_{2}\right) \in \\
&\left.\quad \operatorname{conv}(\mathscr{A}) \text { such that } R \geq R_{1}+R_{2}\right\} \\
& \mathscr{A}_{\text {sum }} \triangleq\left(R, D_{0}, D_{1}, D_{2}\right): \exists\left(R_{1}, R_{2}, D_{0}, D_{1}, D_{2}\right) \in \mathscr{A} \\
&\left.\quad \text { such that } R \geq R_{1}+R_{2}\right\} \\
& R_{\text {sum }}\left(D_{0}, D_{1}, D_{2}\right) \triangleq \\
& \quad \inf \left\{R:\left(R, D_{0}, D_{1}, D_{2}\right) \in \mathscr{A}_{\text {sum }}\right\}
\end{aligned}
$$

The following result follows directly from the above definitions.

Proposition 1. $\operatorname{conv}\left(\mathscr{A}_{\text {sum }}\right)=\widetilde{\mathscr{A}}_{\text {sum }}$.

Let $f\left(x_{0}, x_{1}, x_{2}, x\right), q\left(x_{0}, x_{1}, x_{2}\right), r\left(x_{1}\right)$, and $s\left(x_{2}\right)$ be functions over $\left(\prod_{i=0}^{i=2} \mathcal{X}_{i}\right) \times \mathcal{X}, \prod_{i=0}^{i=2} \mathcal{X}_{i}, \mathcal{X}_{1}$, and $\mathcal{X}_{2}$, respectively, where all the alphabets are assumed to be finite.
Define

$$
\begin{array}{r}
\mathscr{G}\left(f\left(x_{0}, x_{1}, x_{2}, x\right), q\left(x_{0}, x_{1}, x_{2}\right), r\left(x_{1}\right), s\left(x_{2}\right)\right) \\
\triangleq \sum_{x \in \mathcal{X}} \sum_{x_{0} \in \mathcal{X}_{0}} \sum_{x_{1} \in \mathcal{X}_{1}} \sum_{x_{2} \in \mathcal{X}_{2}} f\left(x_{0}, x_{1}, x_{2}, x\right) \times \\
\log \frac{f\left(x_{0}, x_{1}, x_{2}, x\right)}{p_{X}(x) q\left(x_{0}, x_{1}, x_{2}\right) r\left(x_{1}\right) s\left(x_{2}\right)},
\end{array}
$$

and

$$
\begin{aligned}
\mathscr{P} \triangleq\{ & \left(\tilde{q}\left(x_{0}, x_{1}, x_{2}\right), \tilde{r}\left(x_{1}\right), \tilde{s}\left(x_{2}\right)\right): \tilde{q}\left(x_{0}, x_{1}, x_{2}\right), \tilde{r}\left(x_{1}\right), \text { and } \\
& \tilde{s}\left(x_{2}\right) \text { are nonnegative functions defined over } \\
& \mathcal{X}_{0} \times \mathcal{X}_{1} \times \mathcal{X}_{2}, \mathcal{X}_{1}, \text { and } \mathcal{X}_{2}, \text { respectively; } \\
& \sum_{x_{1} \in \mathcal{X}_{1}} \tilde{r}\left(x_{1}\right)=1 ; \sum_{x_{2} \in \mathcal{X}_{2}} \tilde{s}\left(x_{2}\right)=1 ; \\
& \left.\sum_{x_{0} \in \mathcal{X}_{0}} \tilde{q}\left(x_{0}, x_{1}, x_{2}\right)=1, \forall\left(x_{1}, x_{2}\right) \in \mathcal{X}_{1} \times \mathcal{X}_{2} \cdot\right\} .
\end{aligned}
$$

Proposition 2. $R_{\text {sum }}\left(D_{0}, D_{1}, D_{2}\right)$ is given by (5).

We use $\left(f^{*}\left(x_{0}, x_{1}, x_{2}, x\right), q^{*}\left(x_{0}, x_{1}, x_{2}\right), r^{*}\left(x_{1}\right), s^{*}\left(x_{2}\right)\right)$ to denote the minimizer of the optimization problem given by $(5)$.

Proof of Proposition 2: It can be shown by using the log-sum inequality [5, Theorem 2.7.1] that

$$
\left\{\begin{array}{l}
q^{*}\left(x_{0}, x_{1}, x_{2}\right)=\frac{\sum_{x} f^{*}\left(x_{0}, x_{1}, x_{2}, x\right)}{\sum_{x, x_{0}} f^{*}\left(x_{0}, x_{1}, x_{2}, x\right)}, \\
r^{*}\left(x_{1}\right)=\sum_{x, x_{0}, x_{2}} f^{*}\left(x_{0}, x_{1}, x_{2}, x\right), \\
s^{*}\left(x_{2}\right)=\sum_{x, x_{0}, x_{1}} f^{*}\left(x_{0}, x_{1}, x_{2}, x\right),
\end{array}\right.
$$

which, together with (2), implies (5).

According to the Karush-Kuhn-Tucker (KKT) conditions [15, Proposition 3.3.7] for the minimization problem in (5), there exist $\lambda_{0}, \lambda_{1}, \lambda_{2} \geq 0$ such that

$$
\begin{aligned}
& f^{*}\left(x_{0}, x_{1}, x_{2}, x\right)= \\
& \frac{p_{X}(x) q^{*}\left(x_{0}, x_{1}, x_{2}\right) r^{*}\left(x_{1}\right) s^{*}\left(x_{2}\right) e^{-\sum_{i=0}^{i=2} \lambda_{i} d\left(x, x_{i}\right)}}{\sum_{x_{0}, x_{1}, x_{2}} q^{*}\left(x_{0}, x_{1}, x_{2}\right) r^{*}\left(x_{1}\right) s^{*}\left(x_{2}\right) e^{-\sum_{i=0}^{i=2} \lambda_{i} d\left(x, x_{i}\right)}} .
\end{aligned}
$$

Define $L\left(\lambda_{0}, \lambda_{1}, \lambda_{2}\right)$ by (8). We then have the following result, whose proof is given in Appendix A.

Proposition 3. Let $\left(\widehat{q}\left(x_{0}, x_{1}, x_{2}\right), \widehat{r}\left(x_{1}\right), \widehat{s}\left(x_{2}\right)\right)$ be the minimizer of (8) for some $\left(\lambda_{0}, \lambda_{1}, \lambda_{2}\right) \geq 0$. Let $\widehat{f}\left(x_{0}, x_{1}, x_{2}, x\right)$ be given by (7) with $q^{*}(\cdot), r^{*}(\cdot), s^{*}(\cdot)$ replaced by $\widehat{q}(\cdot), \widehat{r}(\cdot), \widehat{s}(\cdot)$, respectively. Let $\widehat{D}_{i}=\sum_{x_{0}, x_{1}, x_{2}, x} \widehat{f}\left(x_{0}, x_{1}, x_{2}, x\right) d\left(x, x_{i}\right)$. Then, $(\widehat{f}(\cdot), \widehat{q}(\cdot), \widehat{r}(\cdot), \widehat{s}(\cdot))$ is the minimizer of (5) with $D_{i}=\widehat{D}_{i}, i=0,1,2$. Let $\widehat{R}=\mathscr{G}(\widehat{f}(\cdot), \widehat{q}(\cdot), \widehat{r}(\cdot), \widehat{s}(\cdot))$. Then, $\left(\widehat{R}, \widehat{D}_{0}, \widehat{D}_{1}, \widehat{D}_{2}\right) \in \partial\left(\operatorname{conv}\left(\mathscr{A}_{\text {sum }}\right)\right)$.

According to the EGC theorem, the EGC region is formed by convex combinations of points, each of which corresponds to a joint pmf $f\left(x, x_{0}, x_{1}, x_{2}\right)$ over $\mathcal{X} \times \mathcal{X}_{0} \times \mathcal{X}_{1} \times \mathcal{X}_{2}$. It is shown in [6] that the marginals of $f\left(x, x_{0}, x_{1}, x_{2}\right)$, i.e., $f\left(x_{1}\right)$, $f\left(x_{2}\right), f\left(x_{0} \mid x_{1}, x_{2}\right)$, can be used to design the codebooks. However, in universal coding, the $\operatorname{pmf} f\left(x, x_{0}, x_{1}, x_{2}\right)$ is not known a priori, and the codebooks need to be designed beforehand. To find a universal scheme, it is necessary to reformulate (1) so that the probability distributions for designing the codebooks are decoupled from $f\left(x, x_{0}, x_{1}, x_{2}\right)$. With this in mind, we define $\mathscr{G}(\cdot)$ in (3) and establish its relationship 


$$
\begin{gathered}
R_{\text {sum }}\left(D_{0}, D_{1}, D_{2}\right)=\min _{f(\cdot), q(\cdot), r(\cdot), s(\cdot)} \mathscr{G}\left(f\left(x_{0}, x_{1}, x_{2}, x\right), q\left(x_{0}, x_{1}, x_{2}\right), r\left(x_{1}\right), s\left(x_{2}\right)\right) \\
\text { subject to } \sum_{x, x_{0}, x_{1}, x_{2}} f\left(x_{0}, x_{1}, x_{2}, x\right) d\left(x, x_{i}\right) \leq D_{i}, i=0,1,2 . \\
\quad \sum_{x_{0}, x_{1}, x_{2}} f\left(x_{0}, x_{1}, x_{2}, x\right)=p_{X}(x), \forall x \in \mathcal{X}, \\
\left(q\left(x_{0}, x_{1}, x_{2}\right), r\left(x_{1}\right), s\left(x_{2}\right)\right) \in \mathscr{P}, f\left(x_{0}, x_{1}, x_{2}, x\right) \geq 0 . \\
L\left(\lambda_{0}, \lambda_{1}, \lambda_{2}\right) \triangleq \min _{q(\cdot), r(\cdot), s(\cdot)}-\sum_{x} p_{X}(x) \log \left(\sum_{x_{0}, x_{1}, x_{2}} q\left(x_{0}, x_{1}, x_{2}\right) r\left(x_{1}\right) s\left(x_{2}\right) e^{-\sum_{i=0}^{i=2} \lambda_{i} d\left(x, x_{i}\right)}\right) \\
\operatorname{subject~to}\left(q\left(x_{0}, x_{1}, x_{2}\right), r\left(x_{1}\right), s\left(x_{2}\right)\right) \in \mathscr{P} .
\end{gathered}
$$

with $R_{\text {sum }}\left(D_{0}, D_{1}, D_{2}\right)$ in (5). The relationship between $R_{\text {sum }}\left(D_{0}, D_{1}, D_{2}\right)$ and $\widetilde{\mathscr{A}_{\text {sum }}}$ can be found by combining Propositions 1, 2, and 3 .

\section{Generalizing Natural Type Selection to MDC}

\section{A. The Basic Coding Process}

We first present two 2-channel MDC schemes, which form the basis of the UMDC schemes given in Section IV-C. The schemes presented here are motivated by the EGC scheme [6], though they have several crucial differences. It will be seen that, according to the theoretical results in Section IV-B, these schemes can be viewed as "natural type selectors" for UMDC.

Fig. 1 describes the modules constituting the proposed schemes. The encoder has two alternative forms, depicted in Fig 1(a) and Fig. 1(b), respectively. In Fig. 1(a), for each source vector, the encoder first calls a procedure named SearchWithFixedDistortion. The procedure begins with the codebook generation, using three functions $q(\cdot, \cdot, \cdot), r(\cdot)$, and $s(\cdot)$ which satisfy the following condition:

$$
(q(\cdot, \cdot, \cdot), r(\cdot), s(\cdot)) \in \mathscr{P},
$$

where $\mathscr{P}$ is defined in (4). Then, each possible combination of codewords is examined until the distortion constraints are met. The procedure outputs a triple of codewords and a triple of integers $\left(m_{0}, m_{1}, m_{2}\right)$ indicating the locations of the codewords in the codebooks. Elias's $\delta$-code [7] is then used to convert $m_{0}, m_{1}$, and $m_{2}$ into three binary sequences. The binary sequences corresponding to $m_{1}$ and $m_{2}$ are transmitted in Descriptions 1 and 2, respectively. The binary sequence of $m_{0}$ is split into both descriptions. Fig. 1(b) is similar to Fig. 1(a) except that the encoder calls a procedure named SearchWithFixedWeight, which returns the triple of codewords with the least weighted distortion value in the codebooks.

Fig. 1(c) shows the diagram of a side decoder. The side decoder only decodes a portion of the received bitstream. It first performs integer decoding, then a procedure named SideReconstruction is invoked to reconstruct the codewords. The central decoder is displayed in Fig. 1(d). It performs integer decoding to retrieve $\left(m_{0}, m_{1}, m_{2}\right)$, which was previously returned by SearchWithFixedDistortion/Weight at the encoder. Then it calls a procedure named CentralReconstruction. All the procedures mentioned above are given in Fig. 2.
Fig. 1(a), Fig. 1(c), and Fig. 1(d) constitute the scheme of fixed-distortion*. Fig. 1(b), Fig. 1(c), and Fig. 1(d) constitute the scheme of fixed-weight.

In practice, the random sampling performed in the proposed procedures is implemented with pseudo-random number generators (PNGs), which are shared by the encoder and the decoder. Suppose that SearchWithFixedDistortion or SearchWithFixedWeight is invoked for a source vector $\mathbf{x}$. Let $q(\cdot, \cdot, \cdot)$, $r(\cdot)$, and $s(\cdot)$ be the functions input to the procedure, and let $\left(\mathbf{x}_{0}, \mathbf{x}_{1}, \mathbf{x}_{2}\right)$ be the triple of codewords returned. To ensure that $\mathbf{x}_{i}(i=1,2)$ is reconstructed by side decoding of the $i$-th description, and that $\mathbf{x}_{0}$ is reconstructed by central decoding, it suffices for the decoder to know $q(\cdot, \cdot, \cdot), r(\cdot)$, and $s(\cdot)$. In Section IV-C it will be revealed how the decoder synchronizes its $q(\cdot, \cdot, \cdot), r(\cdot), s(\cdot)$ with those of the encoder. For the time being, we assume that the above condition is satisfied.

\section{B. Theoretical Results}

In this section, we derive several results regarding the schemes given in Section IV-A. We begin with some definitions. Let $w\left(x_{0}, x_{1}, x_{2}\right)$ be a pmf over $\mathcal{X}_{0} \times \mathcal{X}_{1} \times \mathcal{X}_{2}$. Define

$$
\begin{aligned}
& I_{A}\left(p_{X}(x), w\left(x_{0}, x_{1}, x_{2}\right), D_{0}, D_{1}, D_{2}\right) \\
& \triangleq \min _{f(\cdot)} \sum_{x \in \mathcal{X}} \sum_{x_{0} \in \mathcal{X}_{0}} \sum_{x_{1} \in \mathcal{X}_{1}} \sum_{x_{2} \in \mathcal{X}_{2}} f\left(x_{0}, x_{1}, x_{2}, x\right) \times \\
& \log \frac{f\left(x_{0}, x_{1}, x_{2}, x\right)}{p_{X}(x) w\left(x_{0}, x_{1}, x_{2}\right)} \\
& \text { subject to } \sum_{x, x_{0}, x_{1}, x_{2}} f\left(x_{0}, x_{1}, x_{2}, x\right) d\left(x, x_{i}\right) \leq D_{i} \text {, } \\
& i=0,1,2 \text {, } \\
& \sum_{x} f\left(x_{0}, x_{1}, x_{2}, x\right)=w\left(x_{0}, x_{1}, x_{2}\right), \\
& \sum_{x_{0}, x_{1}, x_{2}} f\left(x_{0}, x_{1}, x_{2}, x\right)=p_{X}(x), \forall x \in \mathcal{X}, \\
& f\left(x_{0}, x_{1}, x_{2}, x\right) \geq 0 \text {. }
\end{aligned}
$$

Parallel to [3] and the references therein, we refer to $I_{A}(\cdot)$ as "lower mutual information". If the feasible region of the above optimization problem is empty, we set $I_{A}(\cdot)=+\infty$.

\footnotetext{
*Lines 14-16 in SearchWithFixedDistortion correspond to the situation of coding failure. When this procedure is used, $M$ (see line 1) should be chosen large enough so that the probability of coding failure is negligible.
} 


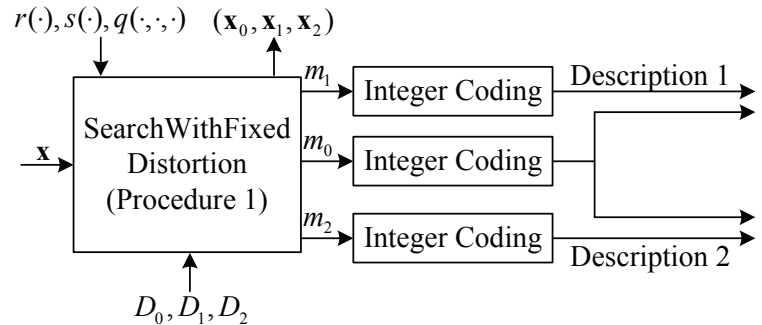

(a) The basic encoder.

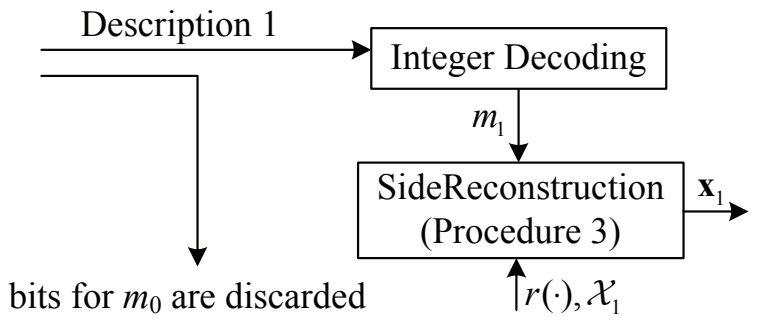

(c) The basic side decoder. The other side decoder has the same structure with $r(\cdot)$ and $\mathcal{X}_{1}$ replaced by $s(\cdot)$ and $\mathcal{X}_{2}$.

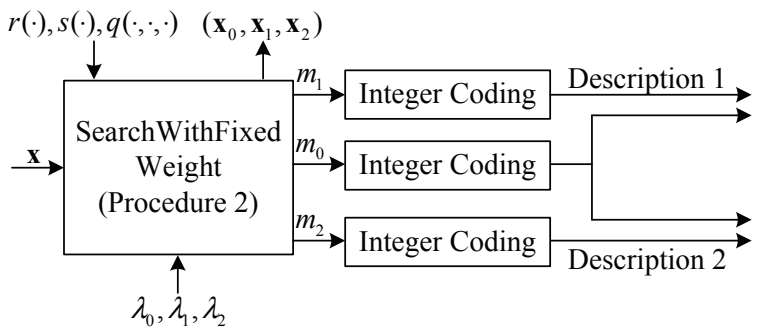

(b) Another form of the basic encoder.

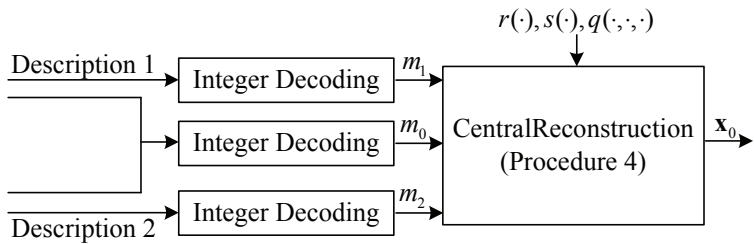

(d) The basic central decoder.

Fig. 1. The basic coding modules. The procedures involved are given in Fig. 2.

For $q\left(x_{0}, x_{1}, x_{2}\right), r\left(x_{1}\right)$, and $s\left(x_{2}\right)$ satisfying (9), define

$$
\begin{aligned}
& \mathscr{D}\left(w\left(x_{0}, x_{1}, x_{2}\right) \| q\left(x_{0}, x_{1}, x_{2}\right) r\left(x_{1}\right) s\left(x_{2}\right)\right) \\
& \quad \triangleq \sum_{x_{0}, x_{1}, x_{2}} w\left(x_{0}, x_{1}, x_{2}\right) \log \left(\frac{w\left(x_{0}, x_{1}, x_{2}\right)}{q\left(x_{0}, x_{1}, x_{2}\right) r\left(x_{1}\right) s\left(x_{2}\right)}\right)
\end{aligned}
$$

and

$$
\begin{aligned}
& R_{A}\left(p_{X}, q, r, s, D_{0}, D_{1}, D_{2}\right) \\
& \quad \triangleq \min _{w}\left\{I_{A}\left(p_{X}, w, D_{0}, D_{1}, D_{2}\right)+\mathscr{D}(w \| q r s)\right\},
\end{aligned}
$$

where we abbreviate $p_{X}(x), w\left(x_{0}, x_{1}, x_{2}\right), q\left(x_{0}, x_{1}, x_{2}\right)$, $r\left(x_{1}\right), s\left(x_{2}\right)$ to $p_{X}, w, q, r, s$, respectively. Note that $I_{A}(\cdot)$ and $R_{A}(\cdot)$ will be used to analyze SearchWithFixedDistortion. For SearchWithFixedWeight, let $\lambda_{0}, \lambda_{1}, \lambda_{2} \geq 0$ and define

$$
\begin{aligned}
& \quad I_{B}\left(p_{X}, w, \lambda_{0}, \lambda_{1}, \lambda_{2}, \bar{D}\right) \\
& \quad \triangleq \min _{D_{0}, D_{1}, D_{2}} I_{A}\left(p_{X}, w, D_{0}, D_{1}, D_{2}\right), \\
& \quad \text { subject to } \sum_{i=0}^{i=2} \lambda_{i} D_{i} \leq \bar{D}, \\
& \quad R_{B}\left(p_{X}, q, r, s, \lambda_{0}, \lambda_{1}, \lambda_{2}, \bar{D}\right) \\
& \quad \triangleq \min _{w}\left\{I_{B}\left(p_{X}, w, \lambda_{0}, \lambda_{1}, \lambda_{2}, \bar{D}\right)+\mathscr{D}(w \| \text { qrs })\right\} .
\end{aligned}
$$

Using (10), we get

$$
\begin{aligned}
& R_{B}\left(p_{X}, q, r, s, \lambda_{0}, \lambda_{1}, \lambda_{2}, \bar{D}\right) \\
& \quad=\min _{D_{0}, D_{1}, D_{2}} R_{A}\left(p_{X}, q, r, s, D_{0}, D_{1}, D_{2}\right) \\
& \quad \text { subject to } \sum_{i=0}^{i=2} \lambda_{i} D_{i} \leq \bar{D} .
\end{aligned}
$$

The properties of $R_{A}(\cdot)$ and $R_{B}(\cdot)$ are summarized as follows.

Proposition 4. If $q\left(x_{0}, x_{1}, x_{2}\right), r\left(x_{1}\right)$, and $s\left(x_{2}\right)$ are strictly positive, then

(i) The minimizers of (10) and (11) are both unique.

(ii) Both $R_{A}\left(p_{X}, q, r, s, D_{0}, D_{1}, D_{2}\right)$ and $R_{B}\left(p_{X}, q, r, s\right.$, $\left.\lambda_{0}, \lambda_{1}, \lambda_{2}, \bar{D}\right)$ are continuous functions of $p_{X}(\cdot)$ under the norm for $p_{X}(\cdot)$ defined by $\left\|p_{X}(\cdot)\right\|_{1}=$ $\sum_{x \in \mathcal{X}}\left|p_{X}(x)\right|$. (iii) $R_{A}\left(p_{X}, q, r, s, D_{0}, D_{1}, D_{2}\right)$ is continuous in ( $D_{0}$, $\left.D_{1}, D_{2}\right) . R_{B}\left(p_{X}, q, r, s, \lambda_{0}, \lambda_{1}, \lambda_{2}, \bar{D}\right)$ is continuous in $\bar{D}$.

Proof Outline: The first result can be proved following similar steps in [3]. The next two can be verified by using the proof technique in [4, Chapter 2].

For $R_{A}(\cdot)$, we also have the following result, which follows from (5).

Proposition 5. $R_{\mathrm{sum}}\left(D_{0}, D_{1}, D_{2}\right)=\min _{q, r, s} R_{A}\left(p_{X}, q\right.$, $\left.r, s, D_{0}, D_{1}, D_{2}\right)$.

The following proposition can be proved using the definition of $R_{B}(\cdot)$.

Proposition 6. Given $p_{X}, q, r, s, \lambda_{0}, \lambda_{1}, \lambda_{2}$, there exists a $\bar{D}_{\max } \geq 0$ such that

(i) $R_{B}\left(p_{X}, q, r, s, \lambda_{0}, \lambda_{1}, \lambda_{2}, \bar{D}\right)>0, \forall \bar{D}<\bar{D}_{\max }$, and is zero otherwise

(ii) $R_{B}\left(p_{X}, q, r, s, \lambda_{0}, \lambda_{1}, \lambda_{2}, \bar{D}\right)$ is a convex and strictly decreasing function of $\bar{D}$ when $0<\bar{D}<\bar{D}_{\max }$.

Next, we introduce two lemmas, which will be used to prove our main results.

Lemma 1. Let $\mathscr{M}_{k}=\left\{\left(m_{0}, m_{1}, m_{2}\right): m_{0}, m_{1}, m_{2}\right.$ are positive integers, $m_{0} m_{1} m_{2}=k$. $\}$. Then,

$$
\lim _{m \rightarrow \infty} \frac{\log \left(\sum_{k=1}^{k=m}\left|\mathscr{M}_{k}\right|\right)}{\log m}=1
$$

Lemma 2. Suppose that $q\left(x_{0}, x_{1}, x_{2}\right), r\left(x_{1}\right)$, and $s\left(x_{2}\right)$ satisfy the constraint in (9). Let $\mathbf{x}$ be some $n$-dimensional vector output by the source. Let $\mathbf{X}_{0}, \mathbf{X}_{1}$, and $\mathbf{X}_{2}$ be three $n$ dimensional random vectors. The elements of $\mathbf{X}_{1}$ and $\mathbf{X}_{2}$ are drawn i.i.d. from the pmfs $r\left(x_{1}\right)$ and $s\left(x_{2}\right)$, respectively. The $i$-th element of $\mathbf{X}_{0}$, denoted by $\mathbf{X}_{0}[i]$, is generated by random 


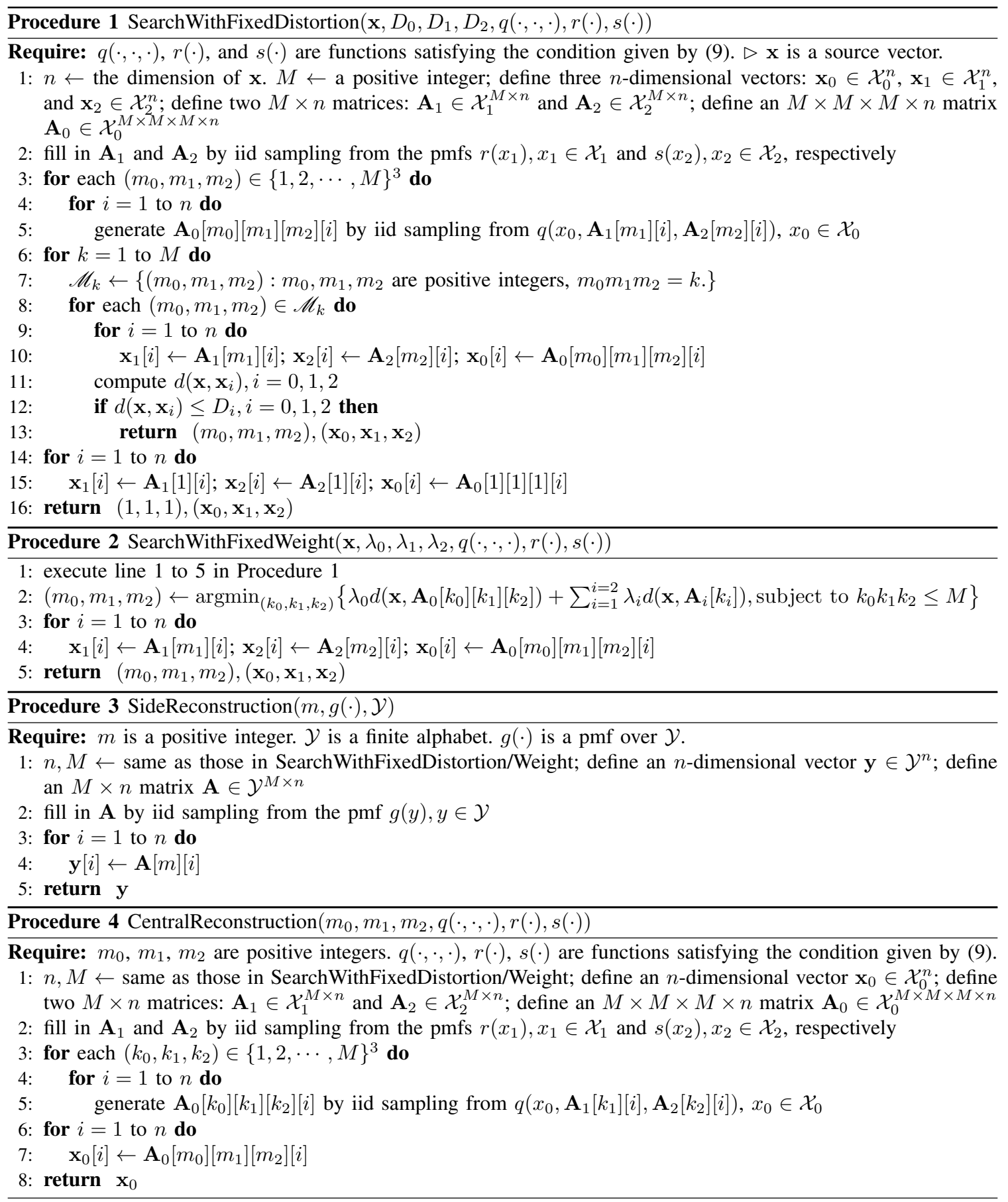

Fig. 2. The procedures which are called by the coding modules given in Fig. 1. $\mathcal{X}_{0}, \mathcal{X}_{1}$, and $\mathcal{X}_{2}$ are finite alphabets. $D_{0}, D_{1}$, and $D_{2}$ are distortions. On line 11 of Procedure $1, d\left(\mathbf{x}, \mathbf{x}_{i}\right)=\frac{1}{n} \sum_{j=1}^{j=n} d_{\mathcal{X}, \mathcal{X}_{i}}\left(\mathbf{x}[j], \mathbf{x}_{i}[j]\right)$.

sampling from the pmf $q\left(x_{0}, \mathbf{X}_{1}[i], \mathbf{X}_{2}[i]\right), x_{0} \in \mathcal{X}_{0}$. Then,

$$
\begin{aligned}
& -\frac{1}{n} \log \left(Q_{A}\left(\mathbf{x}, D_{0}, D_{1}, D_{2}\right)\right) \\
& \quad \triangleq-\frac{1}{n} \log \left(\operatorname{Pr}\left\{d_{\mathcal{X}, \mathcal{X}_{i}}\left(\mathbf{x}, \mathbf{X}_{i}\right) \leq D_{i}, i=0,1,2\right\}\right) \\
& \quad=R_{A}\left(t_{\mathbf{x}}, q, r, s, D_{0}, D_{1}, D_{2}\right)+o(1) \\
& -\frac{1}{n} \log \left(Q_{B}\left(\mathbf{x}, \lambda_{0}, \lambda_{1}, \lambda_{2}, \bar{D}\right)\right) \\
& \quad \triangleq-\frac{1}{n} \log \left(\operatorname{Pr}\left\{\sum_{i=0}^{i=2} \lambda_{i} d\left(\mathbf{x}, \mathbf{X}_{i}\right) \leq \bar{D}\right\}\right) \\
& \quad=R_{B}\left(t_{\mathbf{x}}, q, r, s, \lambda_{0}, \lambda_{1}, \lambda_{2}, \bar{D}\right)+o(1)
\end{aligned}
$$

where $\lim _{n \rightarrow \infty} o(1)=0$.
The proof of Lemma 1 is given in Appendix B. Lemma 2 follows in similar lines to the derivations in [9] and [3]. Equipped with the above lemmas as well as the properties of $R_{A}(\cdot)$ and $R_{B}(\cdot)$, we are ready to analyze the procedures shown in Fig. 2. Our next result reveals how $R_{A}\left(p_{X}, q, r, s, D_{0}, D_{1}, D_{2}\right)$ is related to SearchWithFixedDistortion. It also shows that this procedure plays the role of a "natural type selector".

Proposition 7. Let $\mathbf{X}$ be an $n$-dimensional random source vector. Let 


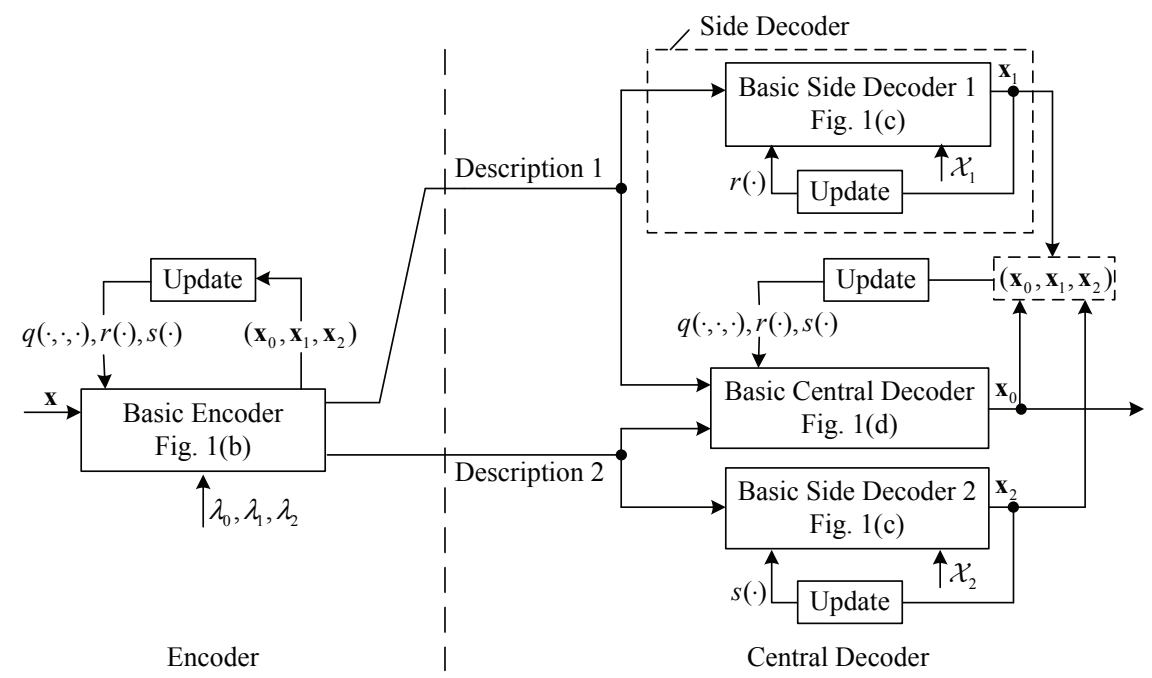

Fig. 3. The complete framework of UMDC.

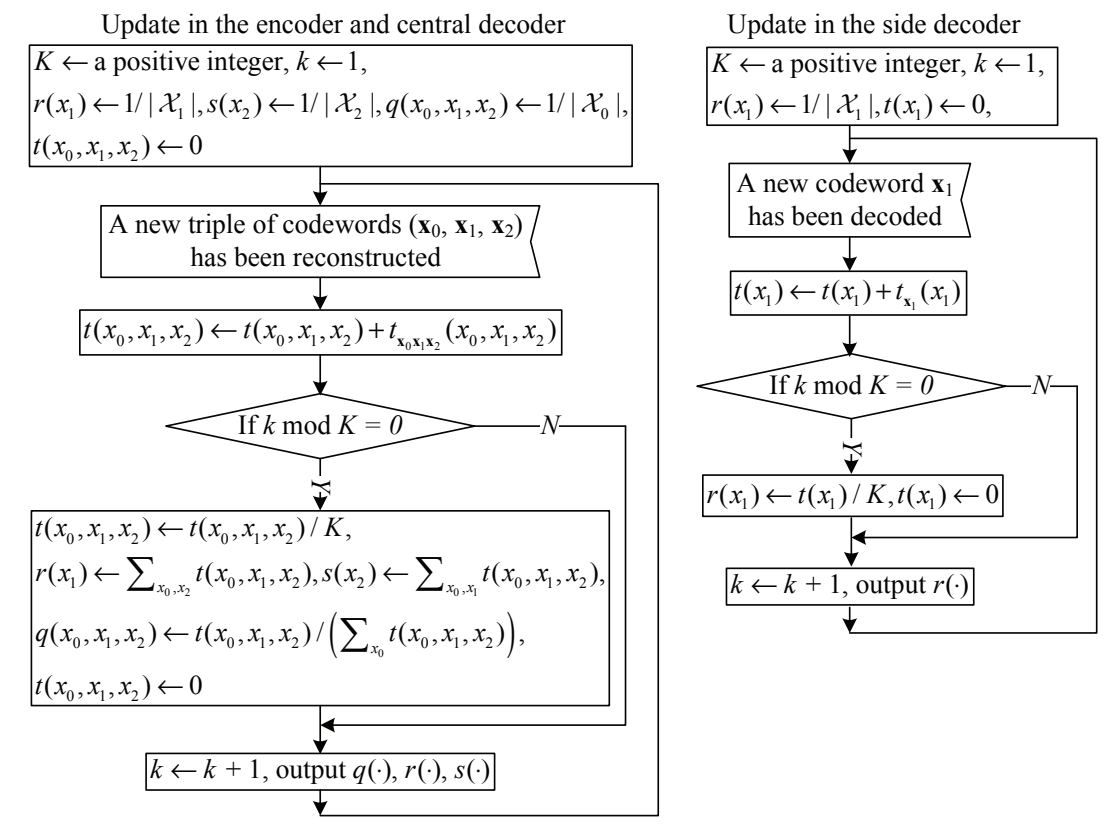

Fig. 4. The update modules in Fig. 3: updating the functions used to generate the codebooks.

$\left(\left(M_{0}, M_{1}, M_{2}\right),\left(\mathbf{X}_{0}, \mathbf{X}_{1}, \mathbf{X}_{2}\right)\right)=$

SearchWithFixedDistortion $\left(\mathbf{X}, D_{0}, D_{1}, D_{2}, q(\cdot, \cdot, \cdot), r(\cdot), s(\cdot)\right)$,

where $\left(M_{0}, M_{1}, M_{2}\right)$ is a triple of positive integers; $\left(\mathbf{X}_{0}, \mathbf{X}_{1}, \mathbf{X}_{2}\right)$ is a triple of $n$-dimensional vectors; $q(\cdot, \cdot, \cdot)$, $r(\cdot)$, and $s(\cdot)$ are positive-valued functions satisfying the constraint in (9). Suppose in SearchWithFixedDistortion, $M \gg$ $2^{n R_{A}\left(p_{X}, q, r, s, D_{0}, D_{1}, D_{2}\right)}$. Then $\forall \epsilon>0, \exists n_{0}>0, \forall n>n_{0}$,

$$
\begin{gathered}
\operatorname{Pr}\left\{\left|\frac{\log \left(M_{0} M_{1} M_{2}\right)}{n}-R_{A}\left(p_{X}, q, r, s, D_{0}, D_{1}, D_{2}\right)\right|<\epsilon\right\} \\
>1-\epsilon \\
\operatorname{Pr}\left\{\| t_{\left.\mathbf{X}_{0} \mathbf{X}_{1} \mathbf{X}_{2}-w_{A} \|_{1}<\epsilon\right\}}>1-\epsilon\right.
\end{gathered}
$$

where $w_{A}$ is the minimizer of (10).
Remark 1. Let $L(\cdot)$ be the length function of Elias's $\delta$-code [7]. It is known [7] that $L(m) \sim \log (m)$. Since Elias's $\delta$ scheme is used to encode each $M_{i}, i=0,1,2$, into a binary sequence, asymptotically the rate of $M_{i}$ is $\frac{1}{n} \log \left(M_{i}\right)$ and the sum-rate equals $\frac{1}{n} \log \left(M_{0} M_{1} M_{2}\right)$. Combining this with (16) and Proposition 5, we note that if $q(\cdot, \cdot, \cdot), r(\cdot)$, and $s(\cdot)$ are chosen appropriately, then asymptotically the coding system depicted in Fig. 1 with Fig. 1(a) as the encoder achieves the EGC sum-rate bound.

Remark 2. By Proposition 4(i), the minimizer of (10) is unique. Thus, (17) implies that the codewords returned by SearchWithFixedDistortion have a joint type approximately equal to $w_{A}$. As a consequence, this procedure can be interpreted as a "type selector".

Remark 3. The conditions given in Proposition 7 for $q(\cdot, \cdot, \cdot)$, $r(\cdot), s(\cdot)$ are slightly stronger than those given by (9). We require $q(\cdot, \cdot, \cdot), r(\cdot), s(\cdot)$ to be strictly positive in order 


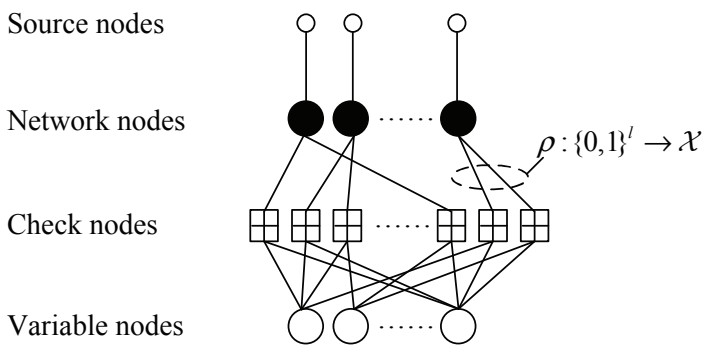

Fig. 5. The multilevel LDGM code proposed in [10].

to prove (16) and (17). But note that to ensure SearchWithFixedDistortion to be executable, the conditions given by (9) suffice. It is unclear whether (16) and (17) hold if $q\left(x_{0}, x_{1}, x_{2}\right) r\left(x_{1}\right) s\left(x_{2}\right)=0$ for some $\left(x_{0}, x_{1}, x_{2}\right)$.

The proof of Proposition 7 is given in Appendix C. For SearchWithFixedWeight, we have the following result, whose proof is given in Appendix D.

Proposition 8 . Let $\mathbf{X}$ be an $n$-dimensional random source vector. Let

$$
\begin{aligned}
& \left(\left(M_{0}, M_{1}, M_{2}\right),\left(\mathbf{X}_{0}, \mathbf{X}_{1}, \mathbf{X}_{2}\right)\right)= \\
& \text { SearchWithFixedWeight }\left(\mathbf{X}, \lambda_{0}, \lambda_{1}, \lambda_{2}, q(\cdot, \cdot, \cdot), r(\cdot), s(\cdot)\right),
\end{aligned}
$$

where $\left(\lambda_{0}, \lambda_{1}, \lambda_{2}\right) \geq 0$ and $q(\cdot, \cdot, \cdot), r(\cdot), s(\cdot)$ are positivevalued functions satisfying the constraint in (9). Suppose in SearchWithFixedWeight, $M=\left\lfloor 2^{n R_{B}\left(p_{X}, q, r, s, \lambda_{0}, \lambda_{1}, \lambda_{2}, \bar{D}\right)}\right\rfloor$ for some $0<\bar{D}<\bar{D}_{\max }$. Then, $\forall \epsilon>0, \exists n_{0}>0, \forall n>n_{0}$,

$$
\begin{gathered}
\operatorname{Pr}\left\{\left|\bar{D}-\sum_{i=0}^{i=2} \lambda_{i} d\left(\mathbf{X}, \mathbf{X}_{i}\right)\right|<\epsilon\right\}>1-\epsilon, \\
\quad \operatorname{Pr}\left\{\| t_{\left.\mathbf{X}_{0} \mathbf{X}_{1} \mathbf{X}_{2}-w_{B} \|_{1}<\epsilon\right\}>1-\epsilon,}\right.
\end{gathered}
$$

where $w_{B}$ is the minimizer of (11).

Remark 4. In view of (19), SearchWithFixedWeight can also be interpreted as a "type selector".

Remark 5. By [7] the sum-rate is asymptotically $\frac{1}{n} \log \left(M_{0} M_{1} M_{2}\right)$. Since $M_{0} M_{1} M_{2} \leq M$, the sum-rate of SearchWithFixedWeight is asymptotically upperbound by $\frac{1}{n} \log (M)$. While SearchWithFixedDistortion returns on the first occurrence of a qualified triple of codewords, SearchWithFixedWeight performs a full search and returns the triple with the minimum weighted distortion. Thus, for SearchWithFixedWeight, we conjecture that $\lim _{n \rightarrow \infty} \frac{1}{n} E\left[\log \left(M_{0} M_{1} M_{2}\right)-\log (M)\right]=0$.

Remark 6. For reasons similar to those given in Remark 3 , we require $q(\cdot, \cdot, \cdot), r(\cdot), s(\cdot)$ to be positive-valued in Proposition 8.

\section{UMDC with Random Codebooks}

Fig. 3 shows the fixed-weight UMDC scheme, whose encoder is built upon Fig. 1(b). The scheme can be divided into two parts: the encoder and the central decoder. The side decoder is a subset of the central decoder and is shown inside a dashed box. Besides the modules given in Fig. 1, the scheme includes several new modules named "Update". Fig. 4 shows how these modules update $q(\cdot), r(\cdot), s(\cdot)$, which are used for codebook generation. With the additional modules,

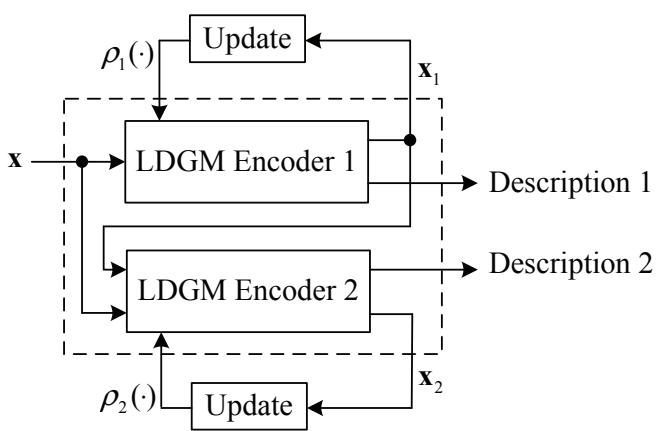

Fig. 6. Updating the codebooks of the MDC scheme proposed in [12] shown inside the dashed box.

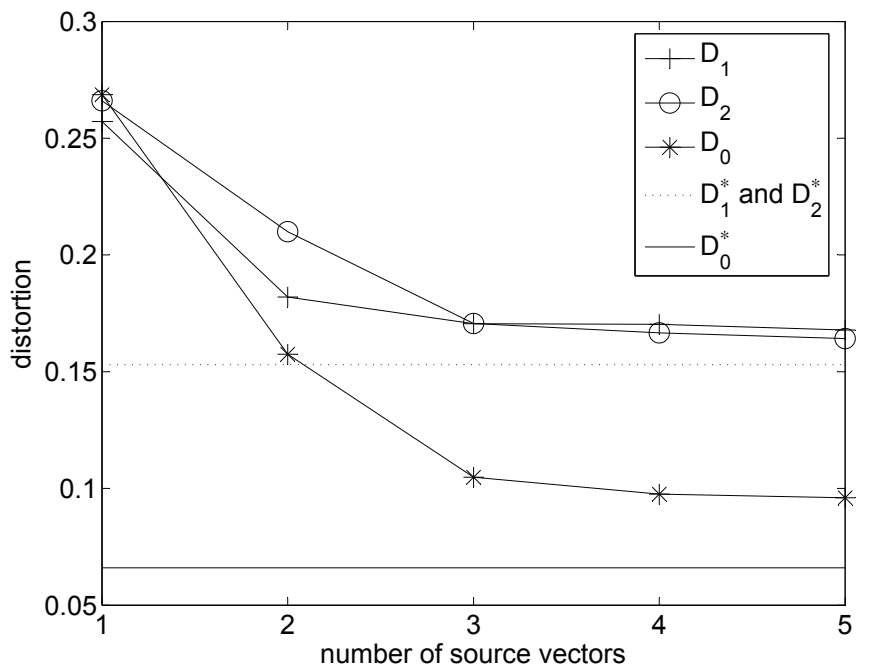

Fig. 7. Convergence behavior of the UMDC scheme on a nonuniform binary source with Hamming distortion. $p_{X}(0)=0.75 . R_{1}=R_{2}=$ $0.231 \mathrm{bit} /$ symbol. $\left(R_{1}, R_{2}, D_{0}^{*}, D_{1}^{*}, D_{2}^{*}\right)$ is located on the EGC bound. The length of each source vector is 10000 .

the decoder is able to synchronize its codebooks with those of the encoder.

Let $\mathbf{X}^{(k)}$ be the $k$-th $n$-dimensional random vector output by the source, and let $\mathbf{X}_{0}^{(k)}, \mathbf{X}_{1}^{(k)}, \mathbf{X}_{2}^{(k)}$ be the reconstruction codewords of $\mathbf{X}^{(k)}$. Moreover, let $\left(q^{(k)}, r^{(k)}, s^{(k)}\right)$ denote the $k$-th triple of functions output by the "Update" module at the encoder. For each $k$, the encoder invokes SearchWithFixedWeight with $\left(q^{(k-1)}, r^{(k-1)}, s^{(k-1)}\right)$ and produces a triple of integers $\left(M_{0}^{(k)}, M_{1}^{(k)}, M_{2}^{(k)}\right)$ indicating the locations of the reconstruction codewords in the codebooks. $\left\{M_{1}^{(k)}: k \geq 1\right\}$ and $\left\{M_{2}^{(k)}: k \geq 1\right\}$ are transmitted in Descriptions 1 and 2, respectively, and $\left\{M_{0}^{(k)}: k \geq 1\right\}$ is split into both descriptions. If there is no transmission error, that is, $\left\{\left(M_{0}^{(k)}, M_{1}^{(k)}, M_{2}^{(k)}\right): k \geq 1\right\}$ is received correctly, then for each $k$, the central decoder could reproduce $\left(q^{(k)}, r^{(k)}, s^{(k)}\right)$ by using its "Update" module given in Fig. 4 with the previously reconstructed codewords. If only one description is received correctly, say the description containing $\left\{M_{1}^{(k)}: k \geq 1\right\}$, then according to Fig. 4, for each $k$ the side decoder could reproduce $r^{(k)}(\cdot)$ with the previously reconstructed codewords $\mathscr{X}_{1}^{(k)}=$ $\left\{\mathbf{X}_{1}^{\left(k^{\prime}\right)}:\lfloor k / K\rfloor K-K+1 \leq k^{\prime} \leq\lfloor k / K\rfloor K\right\}$. Note that the 
function $r^{(k)}(\cdot)$ generated at the encoder depends only on $\mathscr{X}_{1}^{(k)}$. Therefore, the functions required by each decoder for codebook generation can always be obtained from the types of previously reconstructed codewords, and the encoder needs neither to know which description is received, nor to transmit $\left(q^{(k)}, r^{(k)}, s^{(k)}\right)$.

To analyze the R-D performance of the UMDC scheme, we need the following result for $R_{B}(\cdot)$, which can be proved by using the log-sum inequality [5, Theorem 2.7.1].

Proposition 9. Let

$$
\begin{aligned}
\widehat{q}\left(x_{0}, x_{1}, x_{2}\right) & =\frac{w_{B}\left(x_{0}, x_{1}, x_{2}\right)}{\sum_{x_{0}} w_{B}\left(x_{0}, x_{1}, x_{2}\right)}, \\
\widehat{r}\left(x_{1}\right) & =\sum_{x_{0}, x_{2}} w_{B}\left(x_{0}, x_{1}, x_{2}\right), \\
\widehat{s}\left(x_{2}\right) & =\sum_{x_{0}, x_{1}} w_{B}\left(x_{0}, x_{1}, x_{2}\right),
\end{aligned}
$$

where $w_{B}\left(x_{0}, x_{1}, x_{2}\right)$ is the minimizer of (11). Then,

$$
\begin{aligned}
& R_{B}\left(p_{X}, \widehat{q}, \widehat{r}, \widehat{s}, \lambda_{0}, \lambda_{1}, \lambda_{2}, \bar{D}\right) \\
& \quad \leq R_{B}\left(p_{X}, q, r, s, \lambda_{0}, \lambda_{1}, \lambda_{2}, \bar{D}\right) .
\end{aligned}
$$

The following proposition shows that the weighted distortion of the codewords reconstructed by the proposed scheme does not increase with time when $n$ tends to infinity.

Proposition 10. Suppose in SearchWithFixedWeight, $M=$ $\left\lfloor 2^{n C}\right\rfloor$, where $0<C<2 H\left(p_{X}\right)$. Then, $\forall \delta>0, \forall k, \forall \Delta k$,

$$
\begin{array}{r}
\lim _{n \rightarrow \infty} \operatorname{Pr}\left\{\sum_{i=0}^{i=2} \lambda_{i} d\left(\mathbf{X}^{(k+\Delta k)}, \mathbf{X}_{i}^{(k+\Delta k)}\right)\right. \\
\left.-\sum_{i=0}^{i=2} \lambda_{i} d\left(\mathbf{X}^{(k)}, \mathbf{X}_{i}^{(k)}\right)>\delta\right\}=0,
\end{array}
$$

where $\left(\lambda_{0}, \lambda_{1}, \lambda_{2}\right)>0^{\dagger}$.

Proof Outline: For brevity, we assume $K=1$ for the modules given in Fig. 4. The proof of (21) for $K>1$ is similar and is omitted. When $K=1$,

$$
\left\{\begin{aligned}
q^{(k)}\left(x_{0}, x_{1}, x_{2}\right) & =\frac{t^{(k)}\left(x_{0}, x_{1}, x_{2}\right)}{\sum_{x_{0}} t^{(k)}\left(x_{0}, x_{1}, x_{2}\right)}, \\
r^{(k)}\left(x_{1}\right) & =\sum_{x_{0}, x_{2}} t^{(k)}\left(x_{0}, x_{1}, x_{2}\right), \\
s^{(k)}\left(x_{2}\right) & =\sum_{x_{0}, x_{1}} t^{(k)}\left(x_{0}, x_{1}, x_{2}\right),
\end{aligned}\right.
$$

where $t^{(k)}\left(x_{0}, x_{1}, x_{2}\right)$ denotes the joint type of $\left(\mathbf{X}_{0}^{(k)}, \mathbf{X}_{1}^{(k)}, \mathbf{X}_{2}^{(k)}\right)$. Note that if $0<C<2 H\left(p_{X}\right)$, then for any $(q, r, s) \in \mathscr{P}, q, r, s>0$, and $\left(\lambda_{0}, \lambda_{1}, \lambda_{2}\right)>0$, there exists a $\bar{D}>0$ such that

$$
R_{B}\left(p_{X}, q, r, s, \lambda_{0}, \lambda_{1}, \lambda_{2}, \bar{D}\right)=C .
$$

Besides, according to (11), for positive-valued $q(\cdot, \cdot, \cdot)$, $r(\cdot), s(\cdot)$, the minimizer of (11) is also positive-valued. Let $w_{B}^{(k)}\left(x_{0}, x_{1}, x_{2}\right)$ be the minimizer of (11) with $q=$ $q^{(k-1)}, r=r^{(k-1)}, s=s^{(k-1)}$. In view of (19), we have

$$
\lim _{n \rightarrow \infty} \operatorname{Pr}\left\{t^{(k)}=w_{B}^{(k)}\right\}=1 \text {. }
$$

\footnotetext{
${ }^{\dagger}$ Here we assume $\left(\lambda_{0}, \lambda_{1}, \lambda_{2}\right)>0$ instead of $\left(\lambda_{0}, \lambda_{1}, \lambda_{2}\right) \geq 0$ because in the case $\lambda_{0} \lambda_{1} \lambda_{2}=0$ the problem degenerates to successive refinement or single description coding.
}

Combining (23), (22), (20), (18), Proposition 6(ii), and the condition $M=\left\lfloor 2^{n C}\right\rfloor$ with $0<C<2 H\left(p_{X}\right)$, we find that $\forall \delta>0, \forall k$,

$$
\begin{aligned}
\lim _{n \rightarrow \infty} \operatorname{Pr} & \left\{\sum_{i=0}^{i=2} \lambda_{i} d\left(\mathbf{X}^{(k)}, \mathbf{X}_{i}^{(k)}\right)\right. \\
& \left.-\sum_{i=0}^{i=2} \lambda_{i} d\left(\mathbf{X}^{(k-1)}, \mathbf{X}_{i}^{(k-1)}\right)>\delta\right\}=0,
\end{aligned}
$$

from which (21) follows.

One can get the fixed-distortion UMDC scheme by replacing the basic encoder in Fig. 3 with that in Fig. 1(a). It can be shown that as $n$ tends to infinity the sum-rate of this UMDC scheme does not increase with time. It is still an open problem whether the R-D performance of the proposed scheme converges to the EGC bound.

\section{Application to Practical MDC}

We first review the multilevel LDGM code proposed in [10], which is designed for single description coding. Fig. 5 shows the factor graph of a multilevel LDGM code. The code consists of four levels of nodes. Suppose there are $n$ source nodes, $n$ network nodes, and $l \cdot n$ check nodes where $l$ is some integer. Each check node takes binary values. Each network node is connected to one source node and $l$ check nodes, and its value is determined by the values of its adjacent check nodes through a mapping $\rho:\{0,1\}^{l} \rightarrow \hat{\mathcal{X}}$. The reconstruction codeword $\hat{\mathbf{x}} \in \hat{\mathcal{X}}^{n}$ is formed by putting the values of all the network nodes into a vector.

Suppose $\hat{\mathcal{X}}=\mathcal{X}=\{1,2, \cdots,|\mathcal{X}|\}$. Note that according to [5]

$$
\begin{aligned}
R(D) & =\min _{p_{\hat{\mathcal{X}} \mid \mathcal{X}}(\hat{x} \mid x)}\{I(X ; \hat{X}): E[d(X, \hat{X})] \leq D\} \\
& =\mathscr{D}\left(p_{\hat{\mathcal{X}} \mid \mathcal{X}}^{*}(\hat{x} \mid x) p_{X}(x) \| p_{\hat{\mathcal{X}}}^{*}(\hat{x}) p_{X}(x)\right),
\end{aligned}
$$

where $R(D)$ is the R-D function of $p_{X}(x)$. It is shown in [10] that the following mapping is an asymptotically optimal choice for $\rho(\cdot)$ :

$$
\begin{aligned}
& \rho\left(c_{1}, c_{2}, \cdots, c_{l}\right) \\
& =\left\{\begin{array}{l}
\hat{x} \text { if } \sum_{\hat{y}=1}^{\hat{y}=\hat{x}-1}\left\lfloor 2^{l} p_{\hat{\mathcal{X}}}^{*}(\hat{y})\right\rfloor \leq \sum_{k=1}^{k=l} 2^{k-1} c_{k}<\sum_{\hat{y}=1}^{\hat{y}=\hat{x}}\left\lfloor 2^{l} p_{\hat{\mathcal{X}}}^{*}(\hat{y})\right\rfloor, \\
1 \text { if } \sum_{k=1}^{k=l} 2^{k-1} c_{k} \geq \sum_{\hat{y}=1}^{\hat{y}=|\mathcal{X}|}\left\lfloor 2^{l} p_{\hat{\mathcal{X}}}^{*}(\hat{y})\right\rfloor .
\end{array}\right.
\end{aligned}
$$

Denote by $\mathbf{G}$ the generator matrix of the LDGM code, and by $\mathbf{c}$ the vector composed of the values of the check nodes. Note that $\mathbf{c}$ is of dimension $n l$ and $\mathbf{c}=\mathbf{v G}$ for some $\mathbf{v}$. Suppose that the entries of $\mathbf{G}$ are generated randomly and independently of each other with a Bernoulli distribution. Then, $t_{\mathbf{c}}$ approaches the uniform distribution as $n \rightarrow \infty$ for almost all choices of $\mathbf{v}$ (see [10]). With the $\rho(\cdot)$ given by (25), $t_{\hat{\mathbf{x}}}$, the type of the reconstruction codeword, approaches $p_{\hat{\mathcal{X}}}^{*}(\hat{x})$ as $l, n \rightarrow \infty$, which, according to (24), is necessary for the asymptotic optimality of the whole LDGM code.

In [12], a two-channel MDC scheme using the above LDGM code is proposed for binary sources with Hamming 
distortion. The scheme employs two LDGM codebooks, one for each description, and the descriptions are generated sequentially. Given an $n$-dimensional source vector $\mathbf{x}$, the first description is produced with the penalties assigned according to the source symbols. The penalties for the second description is given based on the first description as well as the source vector. Denote by $\mathbf{x}_{j}(j=1,2)$ the side reconstruction codeword generated with the $j$-th description. The central reconstruction codeword $\mathbf{x}_{0}$ is given by the following equation [12]:

$$
\mathbf{x}_{0}[i]=\left\{\begin{array}{l}
0 \text { if } \mathbf{x}_{1}[i]=\mathbf{x}_{2}[i]=0 \\
1 \text { otherwise }
\end{array}\right.
$$

where $1 \leq i \leq n$.

Denote by $\rho_{j}:\{0,1\}^{l_{j}} \rightarrow \mathcal{X}_{j}, j=1,2$, the nonlinear mapping used in the LDGM codebook of the $j$-th description. Based on the MDC scheme proposed in [12], a practical universal scheme is given in Fig. 6. Each time a source vector is encoded into two descriptions, and $\rho_{j}(\cdot)$ is updated according to the following equation:

$$
\rho_{j}\left(c_{1}, c_{2}, \cdots, c_{l_{j}}\right)=\left\{\begin{array}{l}
0 \text { if } \sum_{k=1}^{k=l_{j}} 2^{k-1} c_{k}<\left\lfloor 2^{l_{j}} t_{\mathbf{x}_{j}}(0)+0.5\right\rfloor \\
1 \text { otherwise. }
\end{array}\right.
$$

The practical universal scheme given above only contains two codebooks and does not generate refinement information, which is a simplified version of the scheme given in Fig. 3.

\section{EXPERIMENTAL RESULTS}

We implement the UMDC scheme given in Fig. 6. Fig. 7 shows the convergence behavior of the scheme when applied to a nonuniform binary source with Hamming distortion. We set the length of each source vector to 10000 , and set $l_{1}=l_{2}=4$. The generator matrices of the LDGM codes are designed using the algorithm given in [13] for BIAWGN (Binary Input Additive White Gaussian Noise) channels. For the message passing and decimation over the LDGM codes, the corresponding parameters are chosen according to [10] and [12]. The central decoder combines both descriptions with (26). In the "Update" modules depicted in Fig. 4, we set $K=1$. The curves in Fig. 7 are averaged over 5 independent trials. In Fig. 7, it is observed that the UMDC scheme converges quickly. Initially, the central distortion is slightly larger than the side distortions, because the central decoder always combines both descriptions with (26) and the codebooks at that time are far from being optimal. However, as more source vectors are coded, the central distortion decreases faster, and is eventually less than the side distortions. Tab. I compares the performance of the universal scheme (when it converges) with the non-universal scheme proposed in [11] and the EGC bound in the no-excess sum rate case [14]. On average, the central and side distortions of the proposed scheme are comparable with those of the non-universal scheme, and are about 0.022 , 0.010, 0.007 larger than those given by the EGC theorem, respectively.

\section{CONCLUSIONS}

We consider universal coding in the context of multiple descriptions. We propose two UMDC schemes using random codebooks. The principle of natural type selection is generalized to MDC and the supporting theoretical results are proved. It is shown that in each scheme the types of reconstruction codewords can help to improve the R-D performance. We then give a practical universal scheme based on the LDGM-based MDC methods. The practical scheme derives its principles from our theoretical framework. Each time a source vector is encoded, the nonlinear mappings inside the LDGM codes are updated according to the types of reconstruction codewords. Experimental results show that the R-D performance of the schemes gradually approaches the EGC bound.

\section{ACKNOWLEDGEMENT}

The authors would like to thank the Associate Editor and the two anonymous reviewers for their valuable comments and suggestions.

\section{APPENDIX A}

Proof of Proposition 3: Given $\lambda_{0}, \lambda_{1}, \lambda_{2} \geq 0$, define

$$
\begin{gathered}
\mathscr{L}\left(f\left(x_{0}, x_{1}, x_{2}, x\right), q\left(x_{0}, x_{1}, x_{2}\right), r\left(x_{1}\right), s\left(x_{2}\right), \lambda_{0}, \lambda_{1}, \lambda_{2}\right) \\
\triangleq \\
\quad \mathscr{G}\left(f\left(x_{0}, x_{1}, x_{2}, x\right), q\left(x_{0}, x_{1}, x_{2}\right), r\left(x_{1}\right), s\left(x_{2}\right)\right) \\
\quad+\sum_{i=0}^{i=2} \sum_{x_{0}, x_{1}, x_{2}, x} \lambda_{i} f\left(x_{0}, x_{1}, x_{2}, x\right) d\left(x, x_{i}\right) .
\end{gathered}
$$

For functions $f\left(x_{0}, x_{1}, x_{2}, x\right), q\left(x_{0}, x_{1}, x_{2}\right), r\left(x_{1}\right), s\left(x_{2}\right)$ satisfying the constraints in (5) with $D_{i}=\widehat{D}_{i}$, we have

$$
\begin{aligned}
& \mathscr{L}\left(\widehat{f}, \widehat{q}, \widehat{r}, \widehat{s}, \lambda_{0}, \lambda_{1}, \lambda_{2}\right) \\
& \quad=L\left(\lambda_{0}, \lambda_{1}, \lambda_{2}\right) \leq \mathscr{L}\left(f, q, r, s, \lambda_{0}, \lambda_{1}, \lambda_{2}\right) .
\end{aligned}
$$

Since $\sum f\left(x_{0}, x_{1}, x_{2}, x\right) d\left(x, x_{i}\right) \leq \widehat{D}_{i}, i=0,1,2$, it follows that $\mathscr{G}(\widehat{f}, \widehat{q}, \widehat{r}, \widehat{s}) \leq \mathscr{G}(f, q, r, s)$, which means that $(\widehat{f}, \widehat{q}, \widehat{r}, \widehat{s})$ is the minimizer of (5) with $D_{i}=\widehat{D}_{i}$. Notice that the inequality in (27) holds irrespective of the values of $\widehat{D}_{i}, i=0,1,2$. Thus, $\left(\widehat{R}, \widehat{D}_{0}, \widehat{D}_{1}, \widehat{D}_{2}\right)$ is located on a supporting hyperplane of $\mathscr{A}_{\text {sum }}$, which implies that it belongs to $\partial\left(\operatorname{conv}\left(\mathscr{A}_{\text {sum }}\right)\right)$.

\section{APPENDIX B}

Proof of Lemma 1: For each positive integer $k$, define $\alpha(k) \triangleq|\{l: l \mid k, l>0\}|$, where " $l \mid k$ " stands for " $l$ divides $k$ ". Then

$$
\sum_{k=1}^{k=m}\left|\mathscr{M}_{k}\right|=\sum_{k=1}^{k=m} \sum_{l \mid k} \alpha(l)=\sum_{k=1}^{k=m}\left\lfloor\frac{m}{k}\right\rfloor \alpha(k) .
$$

Thus,

$$
m \sum_{k=1}^{k=m} \frac{\alpha(k)}{k}-\sum_{k=1}^{k=m} \alpha(k) \leq \sum_{k=1}^{k=m}\left|\mathscr{M}_{k}\right| \leq m \sum_{k=1}^{k=m} \frac{\alpha(k)}{k} .
$$


TABLE I

COMPARISON OF THE R-D PERFORMANCE OF THE PROPOSED UMDC SCHEME (3RD COLUMN), THE NON-UNIVERSAL SCHEME [11] (4TH COLUMN), AND THE EGC BOUND IN THE NO-EXCESS-RATE CASE [14] (5TH COLUMN) FOR BINARY SOURCES WITH HAMMING DISTORTION. THE UNIT OF RATE IS BIT/SYMBOL.

\begin{tabular}{|c|c|c|c|c|}
\hline$p_{X}(0)$ & $\left(R_{1}, R_{2}\right)$ & $\left(D_{0}, D_{1}, D_{2}\right)(\mathrm{UMDC})$ & $\left(D_{0}, D_{1}, D_{2}\right)[11]$ & $\left(D_{0}^{*}, D_{1}^{*}, D_{2}^{*}\right)$ \\
\hline 0.5 & $(0.320,0.320)$ & $(0.091,0.236,0.275)$ & $(0.088,0.235,0.284)$ & $(0.068,0.230,0.264)$ \\
\hline 0.5 & $(0.231,0.231)$ & $(0.157,0.286,0.293)$ & $(0.149,0.275,0.318)$ & $(0.124,0.273,0.287)$ \\
\hline 0.5 & $(0.395,0.395)$ & $(0.052,0.205,0.254)$ & $(0.050,0.206,0.270)$ & $(0.033,0.203,0.251)$ \\
\hline 0.5 & $(0.167,0.628)$ & $(0.047,0.345,0.135)$ & $(0.046,0.333,0.150)$ & $(0.032,0.332,0.134)$ \\
\hline 0.75 & $(0.320,0.320)$ & $(0.048,0.137,0.145)$ & $(0.050,0.139,0.148)$ & $(0.026,0.125,0.136)$ \\
\hline 0.75 & $(0.231,0.231)$ & $(0.096,0.168,0.164)$ & $(0.088,0.162,0.166)$ & $(0.066,0.153,0.153)$ \\
\hline 0.75 & $(0.395,0.395)$ & $(0.021,0.119,0.134)$ & $(0.023,0.121,0.137)$ & $(0.002,0.110,0.124)$ \\
\hline 0.75 & $(0.167,0.628)$ & $(0.016,0.185,0.068)$ & $(0.018,0.188,0.069)$ & $(0.002,0.178,0.060)$ \\
\hline
\end{tabular}

According to $[8, \mathrm{p} .57, \mathrm{p} .70]$,

$$
\begin{aligned}
& \sum_{\substack{k=1 \\
k=m}}^{k=m} \frac{\alpha(k)}{k}=\frac{1}{2} \log ^{2}(m)+2 C_{0} \log (m)+O(1), \\
& \sum_{k=1}^{k} \alpha(k)=m \log m+\left(2 C_{0}-1\right) m+O(\sqrt{m}),
\end{aligned}
$$

where $C_{0}$ is the Euler's constant. Then, we have

$$
C_{1} m \log ^{2} m \leq \sum_{k=1}^{k=m}\left|\mathscr{M}_{k}\right| \leq C_{2} m \log ^{2} m
$$

where $C_{1}$ and $C_{2}$ are constants. Hence, (13) is proved.

\section{APPENDIX C}

Proof of Proposition 7: For brevity, we use $R_{A}\left(p_{X}, \delta\right)$ as the shorthand for $R_{A}\left(p_{X}, q, r, s, D_{0}-\delta, D_{1}-\delta, D_{2}-\delta\right)$. To establish (16), we need to prove that $\forall \epsilon, 0<\epsilon<R_{A}\left(p_{X}, 0\right)$, $\exists n_{1}>0, \forall n>n_{1}$,

$$
\operatorname{Pr}\left\{\frac{1}{n} \log \left(M_{0} M_{1} M_{2}\right)<R_{A}\left(p_{X}, 0\right)-\epsilon\right\}<\epsilon / 2,
$$

and that $\forall \epsilon>0, \exists n_{2}, \forall n>n_{2}$,

$$
\operatorname{Pr}\left\{\frac{1}{n} \log \left(M_{0} M_{1} M_{2}\right)>R_{A}\left(p_{X}, 0\right)+\epsilon\right\}<\epsilon / 2 .
$$

We prove (28) by characterizing the size of the codebook used by SearchWithFixedDistortion, and prove (29) by relating it to the set of jointly typical sequences.

We first prove (28). Define $\left.N_{n} \triangleq \mid 2^{n\left(R_{A}\left(p_{X}, 0\right)-\epsilon\right)}\right\rfloor$. In view of Lemma 1, there exists $n_{1}^{\prime}>0$, for all $n>n_{1}^{\prime}$,

$$
\left|\bigcup_{k=1}^{k=N_{n}} \mathscr{M}_{k}\right| \leq 2^{n\left(R_{A}\left(p_{X}, 0\right)-3 \epsilon / 4\right)} .
$$

By Proposition 4(ii), $\exists \xi>0, \forall \operatorname{pmf} t(\cdot)$ over $\mathcal{X}$, if $\| t(\cdot)-$ $p_{X}(\cdot) \|_{1} \triangleq \sum_{x \in \mathcal{X}}\left|t(x)-p_{X}(x)\right|<\xi$,

$$
\left|R_{A}\left(t, q, r, s, D_{0}, D_{1}, D_{2}\right)-R_{A}\left(p_{X}, 0\right)\right|<\epsilon / 4 .
$$

For any finite alphabet $\mathcal{Y}$ and any pmf over it, say $g(y), y \in \mathcal{Y}$, we use $T_{n, \varepsilon}(g)$ to denote the strongly $\varepsilon$-typical set of $g(\cdot)$ in $\mathcal{Y}^{n}[5]$, i.e.,

$$
T_{n, \varepsilon}(g) \triangleq\left\{\mathbf{y} \in \mathcal{Y}^{n}:\left|t_{\mathbf{y}}(y)-g(y)\right|<\varepsilon /|\mathcal{Y}|, \forall y \in \mathcal{Y}\right\} .
$$

Let $\mathbf{X}$ be an $n$-dimensional random source vector. Then, $\exists n_{1}^{\prime \prime}>0$ such that $\forall n>n_{1}^{\prime \prime}$,

$$
\operatorname{Pr}\left\{\mathbf{X} \in T_{n, \xi}\left(p_{X}\right)\right\}>1-\epsilon / 4 .
$$

Note that $T_{n, \xi}\left(p_{X}\right) \subset\left\{\mathbf{x}:\left\|t_{\mathbf{x}}-p_{X}\right\|_{1}<\xi\right\}$. Combining this fact with (14) and (31) implies that $\exists n_{1}^{\prime \prime \prime}>0, \forall n>n_{1}^{\prime \prime \prime}$, $\forall \mathbf{x} \in T_{n, \xi}\left(p_{X}\right)$,

$$
Q_{A}\left(\mathbf{x}, D_{0}, D_{1}, D_{2}\right) \leq 2^{-n\left(R_{A}\left(p_{X}, 0\right)-\epsilon / 2\right)} .
$$

Then, $\forall n>n_{1} \triangleq \max \left\{n_{1}^{\prime}, n_{1}^{\prime \prime}, n_{1}^{\prime \prime \prime}\right\}$,

$$
\begin{aligned}
& \operatorname{Pr}\left\{M_{0} M_{1} M_{2}<2^{n\left(R_{A}\left(p_{X}, 0\right)-\epsilon\right)}\right\} \\
& \quad \leq \frac{\epsilon}{4}+\sum_{\mathbf{x} \in T_{n, \xi}\left(p_{X}\right)}\left|\bigcup_{k=1}^{k=N_{n}} \mathscr{M}_{k}\right| Q_{A}\left(\mathbf{x}, D_{0}, D_{1}, D_{2}\right) \times \\
& \prod_{i=1}^{i=n} p_{X}(\mathbf{x}[i]) \\
& \quad \stackrel{(a)}{\leq} 2^{n\left(R_{A}\left(p_{X}, 0\right)-3 \epsilon / 4\right)} 2^{-n\left(R_{A}\left(p_{X}, 0\right)-\epsilon / 2\right)}+\epsilon / 4 \\
& =2^{-n \epsilon / 4}+\epsilon / 4,
\end{aligned}
$$

where (a) is due to (30) and (32). This proves (28).

Next we prove (29). By Proposition 4(iii), $\forall \epsilon>0, \exists \delta>0$, such that $\left|R_{A}\left(p_{X}, \delta\right)-R_{A}\left(p_{X}, 0\right)\right|<\epsilon / 4$. In view of (10), we have

$$
\begin{aligned}
& R_{A}\left(p_{X}, \delta\right) \\
& =\min _{f(\cdot)} \mathscr{G}(f, q, r, s) \\
& \text { subject to } \sum_{x_{0}, x_{1}, x_{2}} f\left(x_{0}, x_{1}, x_{2}, x\right)=p_{X}(x) \text {, } \\
& f\left(x_{0}, x_{1}, x_{2}, x\right) \geq 0 \text {, } \\
& \sum_{x_{0}, x_{1}, x_{2}, x} f\left(x_{0}, x_{1}, x_{2}, x\right) d\left(x, x_{i}\right) \leq D_{i}-\delta, \\
& i=0,1,2 \text {, }
\end{aligned}
$$

where $\mathscr{G}(f, q, r, s)$ is defined in (3). Let $f_{\delta}^{*}\left(x_{0}, x_{1}, x_{2}, x\right)$ be the minimizer of the above problem. Then,

$$
\sum_{x_{0}, x_{1}, x_{2}, x} f_{\delta}^{*}\left(x_{0}, x_{1}, x_{2}, x\right) d\left(x, x_{i}\right) \leq D_{i}-\delta, i=0,1,2,
$$


which implies that $\exists \eta_{0}>0, \forall \eta, 0<\eta<\eta_{0}, \forall\left(\mathbf{x}_{0}, \mathbf{x}_{1}\right.$, $\left.\mathbf{x}_{2}, \mathbf{x}\right) \in T_{n, \eta}\left(f_{\delta}^{*}\right)$,

$$
\sum_{x, x_{0}, x_{1}, x_{2}} t_{\mathbf{x}_{0}, \mathbf{x}_{1}, \mathbf{x}_{2}, \mathbf{x}}\left(x_{0}, x_{1}, x_{2}, x\right) d\left(x, x_{i}\right) \leq D_{i}-\delta / 2
$$$$
i=0,1,2 \text {. }
$$

Define the following random events:

$$
\begin{aligned}
\mathcal{E}_{1} \triangleq\{ & \exists\left(m_{0}, m_{1}, m_{2}\right), m_{0} m_{1} m_{2} \leq 2^{n\left(R_{A}\left(p_{X}, 0\right)+\epsilon\right)}, \\
& d\left(\mathbf{X}, \mathbf{A}_{i}\left[m_{i}\right]\right) \leq D_{i}, i=1,2, \\
& \left.d\left(\mathbf{X}, \mathbf{A}_{0}\left[m_{0}\right]\left[m_{1}\right]\left[m_{2}\right]\right) \leq D_{0}\right\}, \\
\mathcal{E}_{2} \triangleq\{ & \exists\left(m_{0}, m_{1}, m_{2}\right), m_{0} m_{1} m_{2} \leq 2^{n\left(R_{A}\left(p_{X}, \delta\right)+\epsilon / 2\right)}, \\
& \left.\left(\mathbf{A}_{0}\left[m_{0}\right]\left[m_{1}\right]\left[m_{2}\right], \mathbf{A}_{1}\left[m_{1}\right], \mathbf{A}_{2}\left[m_{2}\right], \mathbf{X}\right) \in T_{n, \eta}\left(f_{\delta}^{*}\right)\right\},
\end{aligned}
$$

where $\mathbf{A}_{i}, i=0,1,2$, are the matrices defined in SearchWithFixedDistortion, and

$$
\begin{aligned}
\mathbf{A}_{i}\left[m_{i}\right] \triangleq & \left(\mathbf{A}_{i}\left[m_{i}\right][1], \mathbf{A}_{i}\left[m_{i}\right][2], \cdots, \mathbf{A}_{i}\left[m_{i}\right][n]\right), i=1,2, \\
\mathbf{A}_{0}\left[m_{0}\right]\left[m_{1}\right]\left[m_{2}\right] & \\
\triangleq & \left(\mathbf{A}_{0}\left[m_{0}\right]\left[m_{1}\right]\left[m_{2}\right][1], \mathbf{A}_{0}\left[m_{0}\right]\left[m_{1}\right]\left[m_{2}\right][2], \cdots,\right. \\
& \left.\quad \mathbf{A}_{0}\left[m_{0}\right]\left[m_{1}\right]\left[m_{2}\right][n]\right) .
\end{aligned}
$$

Due to (34) and the fact that $R_{A}\left(p_{X}, \delta\right)<R_{A}\left(p_{X}, 0\right)+\epsilon / 4$, we have $\operatorname{Pr}\left\{\mathcal{E}_{2}\right\} \leq \operatorname{Pr}\left\{\mathcal{E}_{1}\right\}$. Thus,

$$
\operatorname{Pr}\left\{\frac{1}{n} \log \left(M_{0} M_{1} M_{2}\right)>R_{A}\left(p_{X}, 0\right)+\epsilon\right\} \leq \operatorname{Pr}\left\{\overline{\mathcal{E}}_{1}\right\} \leq \operatorname{Pr}\left\{\overline{\mathcal{E}}_{2}\right\} .
$$

It can be shown by leveraging the proof technique in [6] that $\operatorname{Pr}\left\{\overline{\mathcal{E}}_{2}\right\} \rightarrow 0$ as $n \rightarrow \infty$, which proves (29).

Finally, we prove (17). Suppose there is another procedure SearchWithFixedDistortion' same as SearchWithFixedDistortion except that it does not return until an integer triple $\left(M_{0}^{\prime}, M_{1}^{\prime}, M_{2}^{\prime}\right)$ is found such that

(i) $\left\|t_{\mathbf{A}_{0}\left[M_{0}^{\prime}\right]\left[M_{1}^{\prime}\right]\left[M_{2}^{\prime}\right] \mathbf{A}_{1}\left[M_{1}^{\prime}\right] \mathbf{A}_{2}\left[M_{2}^{\prime}\right]}-w_{A}\right\| \geq \epsilon$,

(ii) $d\left(\mathbf{A}_{1}\left[M_{1}^{\prime}\right], \mathbf{X}\right) \leq D_{1}, d\left(\mathbf{A}_{2}\left[M_{2}^{\prime}\right], \mathbf{X}\right) \leq D_{2}$, $d\left(\mathbf{A}_{0}\left[M_{0}^{\prime}\right]\left[M_{1}^{\prime}\right]\left[M_{2}^{\prime}\right], \mathbf{X}\right) \leq D_{0}$.

Suppose that the codebooks and source inputs of both procedures are the same. Then,

$$
\begin{aligned}
& \operatorname{Pr}\left\{\| t_{\left.\mathbf{X}_{0} \mathbf{X}_{1} \mathbf{X}_{2}-w_{A} \|<\epsilon\right\}}\right. \\
& \quad \geq \operatorname{Pr}\left\{M_{0} M_{1} M_{2}<M_{0}^{\prime} M_{1}^{\prime} M_{2}^{\prime}\right\} \\
& \quad=1-\operatorname{Pr}\left\{M_{0} M_{1} M_{2}=M_{0}^{\prime} M_{1}^{\prime} M_{2}^{\prime}\right\} .
\end{aligned}
$$

Define

$$
\begin{aligned}
& R_{A, \epsilon}\left(p_{X}, \delta\right) \\
& \triangleq \min _{f(\cdot)} \mathscr{G}(f, q, r, s) \\
& \text { subject to }\left\|\sum_{x} f\left(x_{0}, x_{1}, x_{2}, x\right)-w_{A}\left(x_{0}, x_{1}, x_{2}\right)\right\| \geq \epsilon \text {, } \\
& \sum_{x_{0}, x_{1}, x_{2}} f\left(x_{0}, x_{1}, x_{2}, x\right)=p_{X}(x), \\
& f\left(x_{0}, x_{1}, x_{2}, x\right) \geq 0 \text {, } \\
& \sum_{x_{0}, x_{1}, x_{2}, x} f\left(x_{0}, x_{1}, x_{2}, x\right) d\left(x, x_{i}\right) \leq D_{i}-\delta, \\
& i=0,1,2 \text {; }
\end{aligned}
$$

if the feasible region is empty, we define $R_{A, \epsilon}\left(p_{X}, \delta\right) \triangleq+\infty$. We have $R_{A, \epsilon}\left(p_{X}, 0\right)>R_{A}\left(p_{X}, 0\right)$, which is due to the uniqueness of $w_{A}$. Let $\epsilon^{\prime}=\min \left\{\frac{1}{2} \epsilon, \frac{1}{2}\left(R_{A, \epsilon}\left(p_{X}, 0\right)-\right.\right.$ $\left.\left.R_{A}\left(p_{X}, 0\right)\right)\right\}$. By (16),

$$
\lim _{n \rightarrow \infty} \operatorname{Pr}\left\{M_{0} M_{1} M_{2}<2^{n\left(R_{A}\left(p_{X}, 0\right)+\epsilon^{\prime}\right)}\right\}>1-\frac{\epsilon}{2} .
$$

Note that

$$
\begin{aligned}
\operatorname{Pr}\{\| & \left.t_{\mathbf{X}_{0}} \mathbf{X}_{1} \mathbf{X}_{2}-w_{A} \|<\epsilon\right\} \\
\geq & \operatorname{Pr}\left\{M_{0} M_{1} M_{2}<M_{0}^{\prime} M_{1}^{\prime} M_{2}^{\prime}\right\} \\
\geq & \operatorname{Pr}\left\{M_{0} M_{1} M_{2}<2^{n\left(R_{A}\left(p_{X}, 0\right)+\epsilon^{\prime}\right)}\right. \text { and } \\
& \left.M_{0}^{\prime} M_{1}^{\prime} M_{2}^{\prime} \geq 2^{n\left(R_{A, \epsilon}\left(p_{X}, 0\right)-\epsilon^{\prime}\right)}\right\} \\
> & 1-\operatorname{Pr}\left\{M_{0} M_{1} M_{2} \geq 2^{n\left(R_{A}\left(p_{X}, 0\right)+\epsilon^{\prime}\right)}\right\}- \\
& \operatorname{Pr}\left\{M_{0}^{\prime} M_{1}^{\prime} M_{2}^{\prime}<2^{n\left(R_{A, \epsilon}\left(p_{X}, 0\right)-\epsilon^{\prime}\right)}\right\} .
\end{aligned}
$$

Thus, to establish (17), it suffices to prove

$$
\lim _{n \rightarrow \infty} \operatorname{Pr}\left\{M_{0}^{\prime} M_{1}^{\prime} M_{2}^{\prime}<2^{n\left(R_{A, \epsilon}\left(p_{X}, 0\right)-\epsilon^{\prime}\right)}\right\} \leq \frac{\epsilon}{2} .
$$

Let $\left(\mathbf{Y}_{0}, \mathbf{Y}_{1}, \mathbf{Y}_{2}\right)$ be the triple of random vectors such that

(i) $\left(\mathbf{Y}_{0}, \mathbf{Y}_{1}, \mathbf{Y}_{2}\right)$ is independent of $\mathbf{X}$.

(ii) $\left(\mathbf{Y}_{0}[i], \mathbf{Y}_{1}[i], \mathbf{Y}_{2}[i]\right)$ is independent of $\left(\mathbf{Y}_{0}[j], \mathbf{Y}_{1}[j]\right.$, $\left.\mathbf{Y}_{2}[j]\right), \forall i \neq j$.

(iii) $\operatorname{Pr}\left\{\left(\mathbf{Y}_{0}[i], \mathbf{Y}_{1}[i], \mathbf{Y}_{2}[i]\right)=\left(x_{0}, x_{1}, x_{2}\right)\right\}=r\left(x_{1}\right) s\left(x_{2}\right)$ $q\left(x_{0}, x_{1}, x_{2}\right)$.

Since

$$
\begin{aligned}
& \operatorname{Pr}\left\{\left\|t_{\mathbf{Y}_{0} \mathbf{Y}_{1} \mathbf{Y}_{2}}-w_{A}\right\| \geq \epsilon, d\left(\mathbf{Y}_{i}, \mathbf{x}\right) \leq D_{i}, i=0,1,2\right\}
\end{aligned}
$$

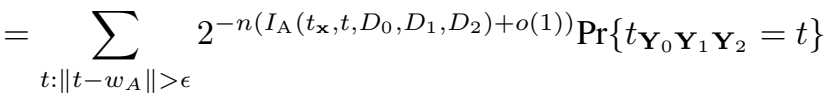

$$
\begin{aligned}
& =2^{-n\left(\min _{w}:\left\|w-w_{A}\right\|>\epsilon\left\{I_{\mathrm{A}}\left(t_{\mathbf{x}}, w, D_{0}, D_{1}, D_{2}\right)+\mathscr{D}(w \| q r s)\right\}+o(1)\right)} \\
& =2^{-n\left(R_{A, \epsilon}\left(t_{\mathbf{x}}, 0\right)+o(1)\right)} \text {, }
\end{aligned}
$$

where the first equality is derived based on the results in [9], and $\underline{\lim }_{p \rightarrow p_{X}} R_{A, \epsilon}(p, 0) \geq R_{A, \epsilon}\left(p_{X}, 0\right)$, there exists $\xi>0$ such that for $n$ sufficiently large,

$$
\begin{aligned}
& \operatorname{Pr}\left\{M_{0}^{\prime} M_{1}^{\prime} M_{2}^{\prime}<2^{n\left(R_{A, \epsilon}\left(p_{X}, 0\right)-\epsilon^{\prime}\right)}\right\} \\
& \leq \frac{\epsilon}{2}+\sum_{\mathbf{x} \in T_{n, \xi}\left(p_{X}\right)}\left(\prod_{i=1}^{i=n} p_{X}(\mathbf{x}[i])\right)\left|\bigcup_{k=1}^{k=2^{n\left(R_{A, \epsilon}\left(p_{X}, 0\right)-\epsilon^{\prime}\right)}} \mathscr{M}_{k}\right| \times \\
& \operatorname{Pr}\left\{\| t_{\mathbf{Y}_{0} \mathbf{Y}_{1} \mathbf{Y}_{2}-w_{A} \| \geq \epsilon,}\right. \\
& \left.\quad d\left(\mathbf{Y}_{i}, \mathbf{x}\right) \leq D_{i}, i=0,1,2\right\} \\
& \stackrel{(a)}{\leq} \frac{\epsilon}{2}+2^{n\left(R_{A, \epsilon}\left(p_{X}, 0\right)-\epsilon^{\prime}+o(1)\right)} 2^{-n\left(R_{A, \epsilon}\left(p_{X}, 0\right)-\frac{\epsilon^{\prime}}{2}+o(1)\right)} \\
& \rightarrow \frac{\epsilon}{2},
\end{aligned}
$$

where (a) follows from Lemma 1. This completes the proof of $(35)^{\ddagger}$.

\section{APPENDIX D}

Proof of Proposition 8: Let $D_{0}, D_{1}, D_{2}$ be values such that $R_{B}\left(p_{X}, q, r, s, \lambda_{0}, \lambda_{1}, \lambda_{2}, \bar{D}\right)=R_{A}\left(p_{X}, q, r, s, D_{0}\right.$,

${ }^{\ddagger}$ If $\varliminf_{p \rightarrow p_{X}} R_{A, \epsilon}(p, 0)=R_{A, \epsilon}\left(p_{X}, 0\right)=+\infty$, then $\exists \eta$, if $\| t_{\mathbf{x}}-$ $p_{X} \| \leq \eta, \operatorname{Pr}\left\{M_{0} M_{1} M_{2}=M_{0}^{\prime} M_{1}^{\prime} M_{2}^{\prime} \mid \mathbf{X}=\mathbf{x}\right\}=0$. 
$\left.D_{1}, D_{2}\right)$ and $\sum_{i=0}^{i=2} \lambda_{i} D_{i} \leq \bar{D} \S$. To prove (18), we only need to prove

$$
\operatorname{Pr}\left\{\sum_{i=0}^{i=2} \lambda_{i} d\left(\mathbf{X}, \mathbf{X}_{i}\right)<\bar{D}-\epsilon\right\}<\epsilon / 2
$$

and

$$
\operatorname{Pr}\left\{\sum_{i=0}^{i=2} \lambda_{i} d\left(\mathbf{X}, \mathbf{X}_{i}\right) \leq \bar{D}+\epsilon\right\}>1-\epsilon / 2,
$$

as $n \rightarrow \infty$. The proof of (36) is similar to that of (28) and is omitted. For (37), note that

$$
\begin{gathered}
\operatorname{Pr}\left\{\sum_{i=0}^{i=2} \lambda_{i} d\left(\mathbf{X}, \mathbf{X}_{i}\right) \leq \bar{D}+\epsilon\right\} \\
\geq \operatorname{Pr}\left\{\exists\left(m_{0}, m_{1}, m_{2}\right), m_{0} m_{1} m_{2} \leq 2^{n R_{A}\left(p_{X}, q, r, s, D_{0}, D_{1}, D_{2}\right)},\right. \\
d\left(\mathbf{X}, \mathbf{A}_{i}\left[m_{i}\right]\right) \leq D_{i}+\frac{\epsilon}{\sum_{i=0}^{i=2} \lambda_{i}}, i=1,2, \\
\left.d\left(\mathbf{X}, \mathbf{A}_{0}\left[m_{0}\right]\left[m_{1}\right]\left[m_{2}\right]\right) \leq D_{0}+\frac{\epsilon}{\sum_{i=0}^{i=2} \lambda_{i}}\right\},
\end{gathered}
$$

which tends to 1 as $n \rightarrow \infty$ according to the proof of (29). Thus, (18) is proved.

We proceed to prove (19). Due to (18), $\forall \delta(0<\delta<\epsilon)$, if $n$ is sufficiently large,

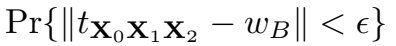

$$
\begin{aligned}
& \geq(1-\delta) \times
\end{aligned}
$$

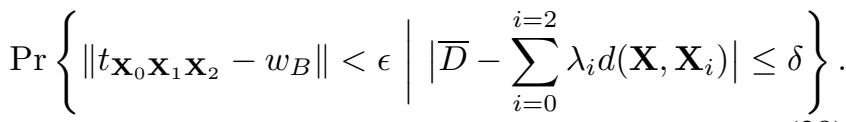

Define

$$
\begin{aligned}
& R_{B, \epsilon}\left(p_{X}, \delta\right) \\
& \triangleq \min _{f(\cdot)} \mathscr{G}(f, q, r, s) \\
& \text { subject to }\left\|\sum_{x} f\left(x_{0}, x_{1}, x_{2}, x\right)-w_{B}\left(x_{0}, x_{1}, x_{2}\right)\right\| \geq \epsilon \text {, } \\
& \sum_{i=0}^{i=2} \sum_{x_{0}, x_{1}, x_{2}, x} f\left(x_{0}, x_{1}, x_{2}, x\right) \lambda_{i} d\left(x, x_{i}\right) \leq \bar{D}+\delta, \\
& \sum_{x_{0}, x_{1}, x_{2}} f\left(x_{0}, x_{1}, x_{2}, x\right)=p_{X}(x), \\
& f\left(x_{0}, x_{1}, x_{2}, x\right) \geq 0,
\end{aligned}
$$

and $R_{B, \epsilon}\left(p_{X}, \delta\right)=+\infty$ if the feasible region is empty. If $\exists \delta_{1}\left(0<\delta_{1}<\epsilon\right)$ such that $R_{B, \epsilon}\left(p_{X}, \delta_{1}\right)=+\infty$, then according to (38), eventually, $\operatorname{Pr}\left\{\left\|t_{\mathbf{X}_{0} \mathbf{X}_{1} \mathbf{X}_{2}}-w_{B}\right\|<\epsilon\right\} \geq$ $1-\delta_{1} \geq 1-\epsilon$, and (19) is proved.

Next we consider the case in which $R_{B, \epsilon}\left(p_{X}, \delta\right)<+\infty$, $0<\delta<\epsilon$. In this case, we actually have $R_{B, \epsilon}\left(p_{X}, \delta\right)<+\infty$, $\forall \delta>0$, since $R_{B, \epsilon}\left(p_{X}, \delta\right)$ is at least non-increasing in $\delta$. Furthermore, by using upper and lower limits, we find that

$$
\lim _{\delta \rightarrow 0^{+}} R_{B, \epsilon}\left(p_{X}, \delta\right)=R_{B, \epsilon}\left(p_{X}, 0\right) .
$$

By Proposition 4(i), $R_{B, \epsilon}\left(p_{X}, \delta\right)>R_{B, 0}\left(p_{X}, \delta\right)=R_{B}\left(p_{X}\right.$, $\left.q, r, s, \lambda_{0}, \lambda_{1}, \lambda_{2}, \bar{D}+\delta\right)$. Thus, (39) implies that $\exists \delta_{2}$

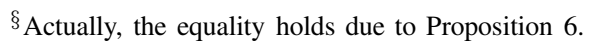

$\left(0<\delta_{2}<\epsilon\right)$ such that $R_{B, \epsilon}\left(p_{X}, 0\right) \geq R_{B, \epsilon}\left(p_{X}, \delta_{2}\right)>$ $R_{B, 0}\left(p_{X}, 0\right)$.

Suppose there is another procedure SearchWithFixedWeight ${ }^{\prime}$ same as SearchWithFixedWeight except that it returns the integer triple $\left(M_{0}^{\prime}, M_{1}^{\prime}, M_{2}^{\prime}\right)$ which satisfies the following equation:

$$
\begin{aligned}
& \left(M_{0}^{\prime}, M_{1}^{\prime}, M_{2}^{\prime}\right) \\
& =\operatorname{argmin}_{\left(k_{0}, k_{1}, k_{2}\right)}\left\{\lambda_{0} d\left(\mathbf{X}, \mathbf{A}_{0}\left[k_{0}\right]\left[k_{1}\right]\left[k_{2}\right]\right)+\right. \\
& \sum_{i=1}^{i=2} \lambda_{i} d\left(\mathbf{X}, \mathbf{A}_{i}\left[k_{i}\right]\right), \\
& \text { subject to } k_{0} k_{1} k_{2} \leq M \text {, } \\
& \left.\left\|t_{\mathbf{A}_{0}\left[k_{0}\right]\left[k_{1}\right]\left[k_{2}\right] \mathbf{A}_{1}\left[k_{1}\right] \mathbf{A}_{2}\left[k_{2}\right]}-w_{B}\right\| \geq \epsilon\right\} .
\end{aligned}
$$

Then,

$$
\begin{aligned}
& \operatorname{Pr}\left\{\| t_{\left.\mathbf{X}_{0} \mathbf{X}_{1} \mathbf{X}_{2}-w_{B} \|<\epsilon\right\}} \geq \operatorname{Pr}\left\{\sum_{i=0}^{i=2} \lambda_{i} d\left(\mathbf{X}, \mathbf{X}_{i}\right)<\lambda_{0} d\left(\mathbf{X}, \mathbf{A}_{0}\left[M_{0}^{\prime}\right]\left[M_{1}^{\prime}\right]\left[M_{2}^{\prime}\right]\right)+\right.\right. \\
& \left.\quad \sum_{i=1}^{i=2} \lambda_{i} d\left(\mathbf{X}, \mathbf{A}_{i}\left[M_{i}^{\prime}\right]\right)\right\} \\
& \geq 1-\operatorname{Pr}\left\{\sum_{i=0}^{i=2} \lambda_{i} d\left(\mathbf{X}, \mathbf{X}_{i}\right) \geq \bar{D}+\frac{\delta_{2}}{2}\right\} \\
& -\operatorname{Pr}\left\{\lambda_{0} d\left(\mathbf{X}, \mathbf{A}_{0}\left[M_{0}^{\prime}\right]\left[M_{1}^{\prime}\right]\left[M_{2}^{\prime}\right]\right)+\sum_{i=1}^{i=2} \lambda_{i} d\left(\mathbf{X}, \mathbf{A}_{i}\left[M_{i}^{\prime}\right]\right)\right. \\
& \left.\quad \leq \bar{D}+\frac{\delta_{2}}{2}\right\} .
\end{aligned}
$$

In view of (37), to prove (19) it suffices to show that

$$
\begin{aligned}
& \lim _{n \rightarrow \infty} \operatorname{Pr}\{ \lambda_{0} d\left(\mathbf{X}, \mathbf{A}_{0}\left[M_{0}^{\prime}\right]\left[M_{1}^{\prime}\right]\left[M_{2}^{\prime}\right]\right)+\sum_{i=1}^{i=2} \lambda_{i} d\left(\mathbf{X}, \mathbf{A}_{i}\left[M_{i}^{\prime}\right]\right) \\
&\left.\leq \bar{D}+\frac{\delta_{2}}{2}\right\}<\epsilon,
\end{aligned}
$$

which can be proved in a way similar to that for (35). Thus, (19) is proved.

\section{REFERENCES}

[1] S. Jalali and T. Weissman, "Multiple description coding of discrete ergodic sources," in Proc. 2009 Allerton Conf. Commun. Contr. Comput., pp. $1256-1261$.

[2] A. György, T. Linder, and G. Lugosi, "Tracking the best quantizer," IEEE Trans. Inf. Theory, vol. 54, no. 4, pp. 1604-1625, Apr. 2008.

[3] R. Zamir and K. Rose, "Natural type selection in adaptive lossy compression," IEEE Trans. Inf. Theory, vol. 47, no. 1, pp. 99-111, Jan. 2001.

[4] I. Csiszár and J. Körner, Information Theory: Coding Theorems for Discrete Memoryless Systems. Akadémiai Kiadó, 1986.

[5] T. M. Cover and J. A. Thomas, Elements of Information Theory. John Wiley \& Sons, Inc., 1991.

[6] A. A. El Gamal and T. M. Cover, "Achievable rates for multiple descriptions," IEEE Trans. Inf. Theory, vol. 28, no. 6, pp. 851-857, Nov. 1982.

[7] P. Elias, "Universal codeword sets and representations of the integers," IEEE Trans. Inf. Theory, vol. 21, pp. 194-203, 1975.

[8] T. M. Apostol, Introduction to Analytic Number Theory. SpringerVerlag, 1976.

[9] Z. Zhang, E. H. Yang, and V. Wei, "The redundancy of source coding with a fidelity criterion-part one: known statistics," IEEE Trans. Inf. Theory, vol. 43, no. 1, pp. 71-91, Jan. 1997.

[10] Z. Sun, M. Shao, J. Chen, K. M. Wong, and X. Wu, "Achieving the rate-distortion bound with low-density generator matrix codes," IEEE Trans. Commun., vol. 58, no. 6, pp. 1643-1653, June 2010.

[11] S. Dumitrescu, J. Chen, and Y. Zhang, "Multiple description coding for binary sources," in Proc. 2009 Int. Conf. Wireless Commun. Signal Process.

[12] Y. Zhang, S. Dumitrescu, J. Chen, and Z. Sun, "Multiple description coding based on LDGM codes," in Proc. 2009 IEEE Inf. Theory Workshop, pp. 85-89. 
[13] S. -Y. Chung, "On the construction of some capacity-approaching coding schemes," Ph.D. dissertation, Massachusetts Institute of Technology, Cambridge, MA, 2000.

[14] R. Ahlswede, "The rate-distortion region for multiple descriptions without excess rate," IEEE Trans. Inf. Theory, vol. 31, pp. 721-726, Nov. 1985.

[15] D. P. Bertsekas, Nonlinear Programming, 2nd edition. Athena Scientific, 1999.

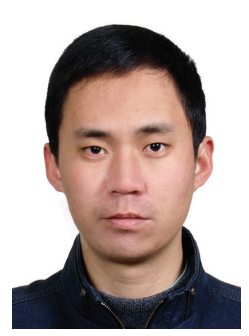

Yuhua Fan received the B.Sc. and M.S. degrees from Shanghai Jiao Tong University, China, in 2005 and 2008, respectively, both in electronic engineering. He is currently pursuing the Ph.D. degree at the same university. His research interests include image and video coding and processing.

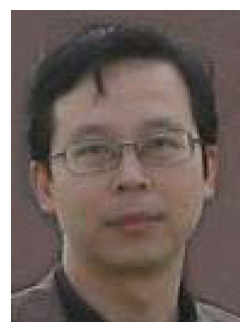

Jia Wang received the B.Sc. degree in electronic engineering, the M.S. degree in pattern recognition and intelligence control, and the Ph.D. degree in electronic engineering from Shanghai Jiao Tong University, China, in 1997, 1999, and 2002, respectively. He is currently an Associate Professor of the Institute of Image Communication and Information Processing, Shanghai Jiao Tong University, and a member of the Shanghai Key Laboratory of Digital Media Processing and Transmission. His research its application in video coding.

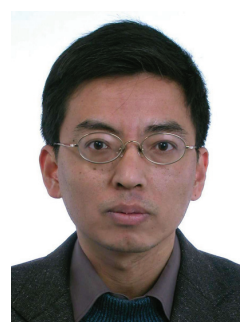

Jun Sun (M'02) received the B.S. and M.S. degrees from the University of Electronic Science and Technology of China, in 1989 and 1992, respectively, and the Ph.D. degree from Shanghai Jiao Tong University, China, in 1995. As a Professor, he is now with the Institute of Image Communication and Information Processing of Shanghai Jiao Tong University. His research interests include image communication, HDTV, and mobile communication.

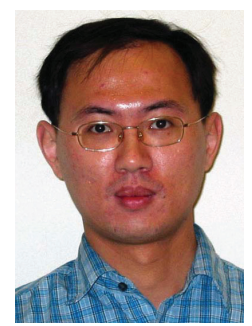

Jun Chen (S'03-M'06) received the B.E. degree with honors in communication engineering from Shanghai Jiao Tong University, Shanghai, China, in 2001 and the M.S. and Ph.D. degrees in electrical and computer engineering from Cornell University, Ithaca, NY, in 2004 and 2006, respectively.

He was a Postdoctoral Research Associate in the Coordinated Science Laboratory at the University of Illinois at Urbana-Champaign, Urbana, IL, from 2005 to 2006, and a Postdoctoral Fellow at the IBM Thomas J. Watson Research Center, Yorktown Heights, NY, from 2006 to 2007. He is currently an Assistant Professor of Electrical and Computer Engineering at McMaster University, Hamilton, ON, Canada. He holds the Barber-Gennum Chair in Information Technology. His research interests include information theory, wireless communications, and signal processing.

He has received several awards for his research, including the Josef Raviv Memorial Postdoctoral Fellowship in 2006, the Early Researcher Award from the Province of Ontario in 2010, and the IBM Faculty Award in 2010. 